International Journal of

Health, Medicine and

Nursing Practice

(IJHMNP)

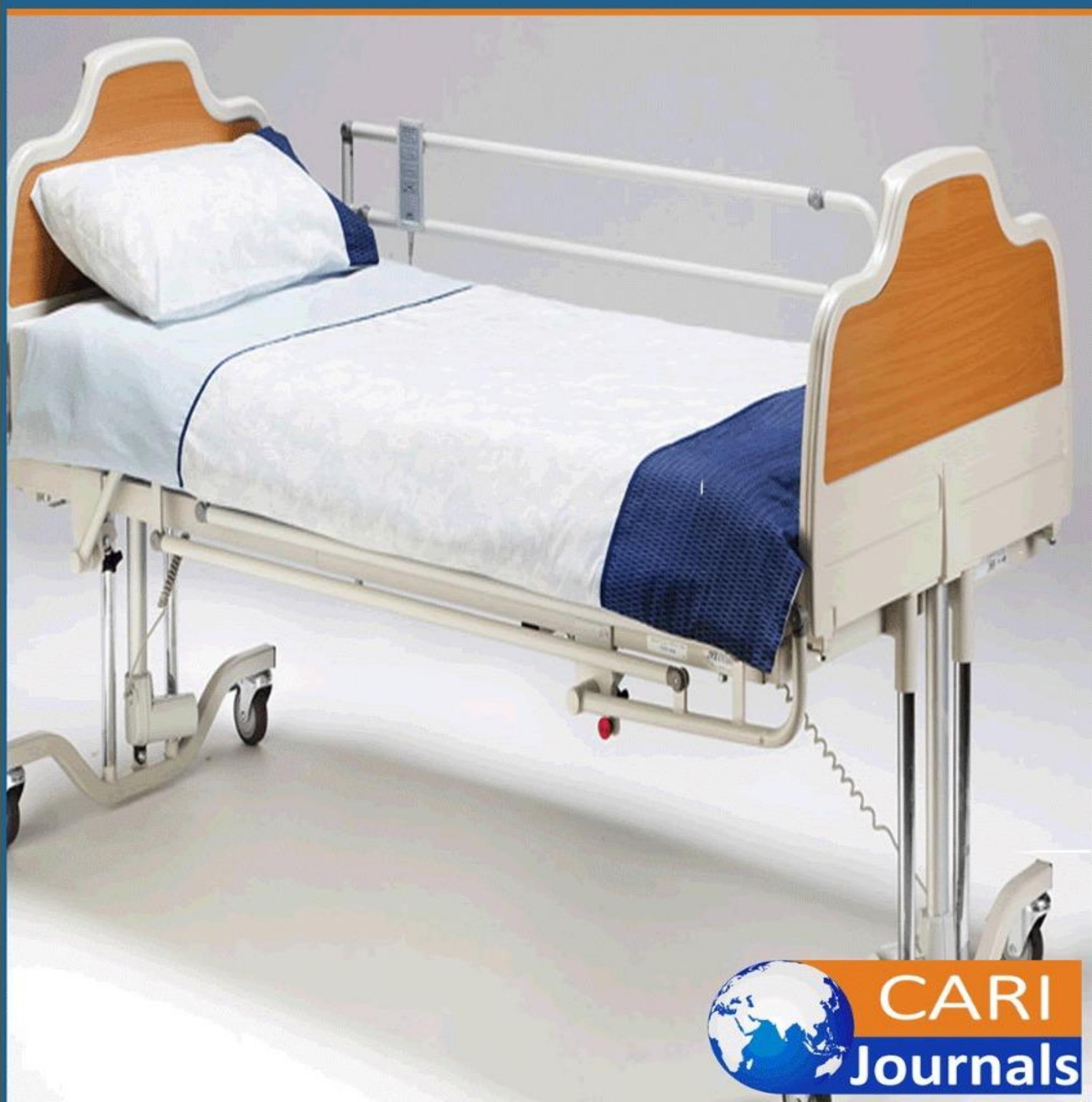


International Journal of Health, Medicine and Nursing Practice

ISSN 2710-1150 (Online)

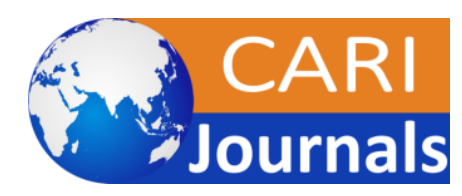

Vol. 3, Issue No. 2, pp 61-106, 2021

www.carijournals.org

\title{
KNOWLEDGE ATTITUDE AND PRACTICES ABOUT ENDOSCOPY PROCEDURES AMONG NURSES OF NISHTAR HOSPITAL MULTAN.
}

\author{
$1^{*}$ Kausar Parveen \\ Clinical Management, Lahore School of Nursing \\ ${ }^{2}$ Mam Kousar Parveen \\ Assistant professor LSN, Lahore School of Nursing \\ *Corresponding Author Email: fahamafnan111@gmail.com
}

\begin{abstract}
Purpose: To assess Knowledge and practices of nurses regarding endoscopic procedures and to identify associated factors towards effective endoscopy procedures

Methodology: A descriptive cross sectional study was designed. The population for this study was 250. There was selection of the population of nurses in Nishtar Hospital Multan. The target population or sample size consists of 150 participants. The nurses were selected from the Nishtar Hospital Multan for the completion of study. Likert scale close ended questionnaire was adopted for data collection from the study participants. Results were analyzed through SPSS.

Results: the nurse age, training and dealing period might have an effect on the level of nurses data relating to canal endoscopy including; general precautions, basic steps to wash and medical care in examination unit. The majority of nurses had positive attitude. While coaching and qualification can affect dealing with the patients additionally to nurses level of apply before, throughout and manual disinfecting of endoscopy.
\end{abstract}

Unique contribution to theory, practice and policy: Extensive training of staff involved in endoscope reprocessing is mandatory for quality assurance and for effective infection control and documentation of this tarring is required. The efficacy of manual cleaning and HLD operator dependent, thus assignment of personnel responsible for endoscope.

Key words: Knowledge, practices of nurses, endoscopy procedures

\section{INTRODUCTION \\ Background of the study}

Endoscopy procedures are much implemented in today's life because demand of such procedures is increasing nowadays. People are facing severe to mild range of gastrointestinal issues and complications. Endoscopy itself is a non-surgical process in which there is thorough examination of human inner digestive tract. This procedure is carried out with the aid of instrument called an endoscope (Alfa MJ et al., 2016). Endoscope is a tube with much flexibility and there is a camera with light attached to this tube. The film or pictures of endoscopy are displayed on specified 
monitor. When there is upper endoscopy, instrument of endoscopy or endoscope is easily entered through mouth region then passed to throat and then reach esophagus (Ali R et al., 2013).

It can continue to stomach and upper region of small intestine of body. Simultaneously, for the purpose of lower endoscopy the endoscopes can be entered from rectum region and pass to the large intestine (colon) region in order to examine this portion of intestinal tract. The basic purpose of endoscopy is to examine whether there is any type of abnormalities in body gastrointestinal tract or not. Moreover, complications like stomach aches, history of gastro ulcers, gastric inflammation, difficulty in swallowing process, bleeding from digestive tract, disturbed bowel movements e.g., severe constipation or diarrhea can leads us to the endoscopy procedures (Bertleff M et al., 2019). There is proper preparation for endoscopy including gut preparation in this type of preparation patient is supposed to undergo an upper digestive tract examination and for this examination there is strict requirement of fasting or NPO (Nil Per Oral) for 6-8 hours before the endoscopic procedure due to the reason that food intake can disturb the entry of endoscope during endoscopy because patient can face reflux when the endoscope is entered through mouth (Carl J, 2019).

In other situation when there is lower endoscopy in order to clear the colon or large intestine patients are given laxatives a day prior to the endoscopy to clear the stool. In addition sedation is also very important for this process hence for most of endoscopic procedures sedative is given to the patient for enhancing the comfort and relaxation of patient. The sedation is given through intravenous route into the veins of body (Cotton PB et al., 2018).

This administered sedation produces relaxation and sleep. Patients usually wake up within an hour after sedation, but side effects of sedatives are more delayed hence it's not safe to use them. In very special conditions type of anesthesia or sedation i.e., General anesthesia is administered which actually puts patient to totally asleep for a period of time it is given in young children, and in situation when very complex procedures are planned according to patients treatments. Gastrointestinal Endoscopy is itself very significant instrument especially for identifying the GI tract disorders and also their treatments (Cowen AE, 2014).

GI endoscopy helps in final diagnosing of several diseases and also aids in allowing minimal application of invasive curable techniques such invasive therapeutic techniques usually replace more aggressive intervention e.g., surgical procedures. Such innovations and advancements gradually reduce mortal rate of people and also minimized hospitalization of patients with GI disorders experiencing treatment procedures (Day T, 2015).

Paramedical staff in any of the setting where GI (gastrointestinal) endoscopy procedures are performed must stick themselves to infection control ethics that will surely create and uphold a safe and secure environment. Such safe environment is actually free from the risk of spreading diseases in patients and the health workers. This is truly despite of the settings like hospitals clinics, ambulatory or mobile care centers and health care offices, all kinds of gastrointestinal (GI) procedures performed in them. Nursing health professionals who continuing work in endoscopy units and providing pre-operative and post-operative care to the patients according to their requirements should have precise training or learning courses. In order to become capable in carrying out their assigned clinical duties in these endoscopy units and also to be able in managing 
the required materials and equipments. So that these all measures can effectively contribute to the optimal success of these treatment procedures (El-Shamaa et al., 2010).

The definite knowledge and the progression of the functions of nurse's goals to develop a secure association with the endoscopy expert to diminish the impediment of the technique implemented, reduction of the patient's possible anxiety, and improvement of the applicability or implementation plus the results of gastrointestinal (GI) endoscopy (Gómez M et al., 2013). Nurses specifically for endoscopy usually plays a critical and significant role in the terms of safe, high quality preparation of endoscopy. Nurses constitute various tasks. For instance preparing the appropriate room for endoscopy, having accurate instrument and necessary equipments or devices is very important for examining the upper or lower Gastrointestinal tract. It is also essential and included in nurse responsibilities that the nurse must provide accurate information about the undergone procedure to the patient, in respect to lessen patient's anxiety. Further to give clarifications regarding the modality of the endoscopic procedure (Kennedy J et al., 2016).

Nurses are called backbone of hospitals and in other healthcare systems. They play vital and effective role in management of several diseases. During endoscopic procedures nurses have strong responsibilities regarding patient's treatment and their cures. However, this important section is still hidden and specifically no researches done on this topic especially in Pakistan Role of nurses and their data collection is absent (Majeski J et al., 2019).

In this high concerned topic research will attempt to perform a KAP study regarding this conceptual topic. Knowledge Attitude and Practices of nurses will be considered in order to highlight the endoscopic treatment and their protocols. This study will have much importance in this regard. The nurses of Nishtar hospital Multan will be study group for the purpose of collecting information and data.

\section{Significance of the study}

The study aimed to provide broad variety of information on endoscopy procedures among nurses of Nishtar Hospital Multan. This study provided appropriate knowledge and practices of nurses in endoscopy procedures. This study improved implementation of nurses and plays an essential role in order to maintain health status of patient with endoscopic procedures and treatments.

\section{Problem of the statement}

A gap aroused between the nurses knowledge and their practices regarding endoscopy procedures and this results in negative impacts or effects on patients with endoscopic complications. Hence their improvement and development is also hindered.

\section{Purpose of the study}

The study provided comprehensive concepts illustrating the knowledge, attitude, and practices regarding endoscopy procedures among nurses in Nishtar Hospital Multan. It helped out to design and implement evidence- based interventions in order to achieve betterment in endoscopic procedures.

\section{Objectives of the study}

\section{General Objectives}


International Journal of Health, Medicine and Nursing Practice

ISSN 2710-1150 (Online)

Vol. 3, Issue No. 2, pp 61-106, 2021

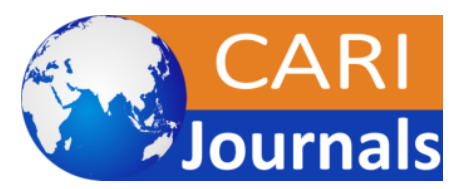

www.carijournals.org

To assess Knowledge and practices of nurses regarding endoscopic procedures and to identify associated factors towards effective endoscopy procedures

\section{Specific Objectives}

To assess Knowledge Attitude and practices of nurses towards effective endoscopic procedures

\section{Research Question}

How to investigate the gap between the current knowledge and actual practices of endoscopy procedures among nurses in Nishtar Hospital Multan.

\section{Operational Definition}

Knowledge is known as awareness or familiarity gained by experience of a fact or situation (Merriam Webster Dictionary, 2018).

Attitude is known as a settled way of thinking or feeling about something (Merriam Webster Dictionary, 2018).

Practice is known as the actual application or use of an idea, belief, or method, as opposed to theories relating to it (Merriam Webster Dictionary, 2018).

Endoscopy is the insertion of a long, thin tube directly into the body to observe an internal organ or tissue in detail. It can also be used to carry out other tasks including imaging and minor surgery (Merriam Webster Dictionary, 2018).

Procedures are a series of actions conducted in a certain order or manner. (Oxford Dictionary, 2020).

\section{Conceptual Definition}

Knowledge is defined as the process of awareness through education on specific topic or subject.

Attitude is defined as thinking and emotional process about something.

Practice is defined as the process of performing theories in particle life

Endoscopy is defined as the process of entering a long tube with camera to visualize the inner body organs clearly.

Procedures are specific protocol of doing something it is also called plan of actions.

\section{LITERATURE REVIEW}

A study was conducted by Wafaa Mohamed Amer, Nadia Mohamed Taha, Howida Kameel Zaton in Medical Surgical Nursing Department, Faculty of Nursing, Zagazig University, Egypt in 2015. All the nurses included in the study were females and their age ranged from 26-58 years. Robinson, Moreau and McCann reported that the common pattern representing nurses' characterized with increased number of female nurse as compared with males. This study revealed that $80 \%$ had of them diploma degrees. More than half of nurses had experience of working in gastrointestinal endoscopy unit range from 8-28 years. Two fifths of them attended training courses during their 
work in GIT Endoscopy and the majority of them had got (HBV) Vaccine. Ramsey and his colleague founded in their study that, most of the nurses did not receive any special education or in- service training about endoscope reprocessing practices. Most of the authors reported that education and training, including competency testing, at least annually. This helps professional nurse to keep up to date on the most recent developments in nursing and to be able to manage the demands of nursing practice. Educational program and training courses are two components of staff development. It is recommended that continuous education in nursing is needed to promote development of knowledge, skills and attitudes of nurses and to improve the quality of care given for their patients. Also the formed training courses played an important role in enhancing and updating nurses' knowledge and performance. There is considerable variation in the field of endoscopy, hence the quality assurance procedures are gaining more significance in the eyes of patients, endoscopists and health care providers. Patient satisfaction questionnaires are a helpful method for detecting opportunities to improve quality based on patient opinion. Assessing patient experiences is thus helpful in identifying areas that require improvement such as the proper delivery of pre-and postprocedural information. Patient satisfaction related to endoscopy procedures is imperative for quality assurance and it may be influenced by various factors as patient characteristics, endoscopists' technique and procedural or organizational features. Numerous societies suggested sedation as a quality indicator for colonoscopy, while the British Society for Gastroenterology and Canadian Association of Gastroenterology associated patient comfort as a supplementary performance indicator. A wide range of well-validated patient comfort scales (varying from generic to colonoscopy specific) are available. One of the most usefull tool is the Nurse-Assessed Patient Comfort Score (NAPCOMS) which was endorsed in the United Kingdom and Canada, being designed in endoscopy units employing minimal to moderate sedation. It records the intensity, frequency, and duration of pain episodes, but it also records the level of sedation and perceived global comfort. In study conducted by Daniela Burtea et al, sedation seemed to play a significant role as a predictor for satisfaction, endoscopy with sedation being well known to reduce anxiety and pain. On the other hand, patients who are committed to cope with un-sedated gastroscopy or colonoscopy described little difference between the pain experienced during the endoscopic examination and pain or anxiety expected before procedure. Several studies outlined that patient satisfaction was associated with the technical abilities of the endoscopist, which was also consistent with our findings. Regarding overall satisfaction or procedure quality as perceived by patients, questionnaires enable the recognition of the most frequent causes for dissatisfaction.

This study revealed that the majority of nurses had satisfactory level of knowledge regarding basic steps to clean and disinfect GI Endoscope. Levels of knowledge among the nurses in the present study about (wearing protective clothes, transferring endoscope for cleaning, pre-manual cleaning stage, test leak, manual cleaning stage, rinsing, sterilization and dryness, dangerous of inadequate endoscope disinfection, storage and documentation). California Department of Health Services (CDHS) stressed up on that nurses need to know how to do it properly without harming themselves by follow the Universal precaution during the procedure. This also was confirmed by Alfa et al.

On the other hand this was not satisfactory for Ramsey and his colleagues, who recommended that continues educational and training guideline program for endoscopies reprocessing will help in effective performance and control infection and they proved this by the significant improvements 
in the post-guidelines program phase. This study revealed that the majority of nurses had a positive attitude towards providing care in endoscopy unit. These results are in agreement with those reported in a survey in the UK, which predicted an important albeit restricted role for nursing endoscopy. Clinicians from the UK considered diagnostic gastro-scopy and sigmoido-scopy appropriate and diagnostic colonoscopy and therapeutic endoscopies inappropriate for NE. The UK audit however did not specifically investigate the attitude towards screening endoscopies.

\section{METHODOLOGY}

\section{Study design}

A descriptive cross sectional study was designed to figure out assessing the knowledge attitudes and practices of nurses regarding endoscopy procedures.

\section{Sample size}

The population for this study was 250 . There was selection of the population of nurses in Nishtar Hospital Multan. The target population or sample size consists of 150 participants.

\section{Study setting}

The nurses were selected from the Nishtar Hospital Multan for the completion of study.

\section{Study Population}

The nurses were selected for the study population

\section{Research tool}

Likert scale close ended questionnaire was adopted for data collection from the study participants. Results were analyzed through SPSS.

\section{Inclusion Criteria}

Inclusion criteria included all nurses of Nishtar Hospital Multan especially who were willing to participate in this study and who gave informed consent.

\section{Exclusion Criteria}

Exclusion criteria included who were not willing to participate in this study. This segment also excluded the absentees' nurses at the time of data collection process.

\section{Research Variables}

\section{Independent Variables}

Knowledge, Attitude and Practices

\section{Dependant Variables}

Endoscopy Procedures

\section{Sample Size}

Slovian's Sampling was used to find the sample size of the study population if the total population is 240 
International Journal of Health, Medicine and Nursing Practice

ISSN 2710-1150 (Online)

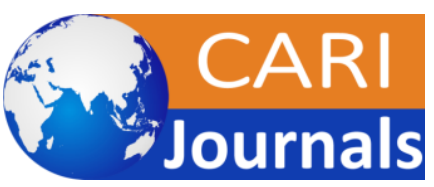

Vol. 3, Issue No. 2, pp 61-106, 2021

www.carijournals.org

If $\mathrm{N}=$ Popoulation $\mathrm{n}=$ sample size $\mathrm{E}=$ Margin of Error

$\mathrm{n}=\mathrm{N} / 1+(\mathrm{N})(\mathrm{E})^{2}$

$\mathrm{n}=240 / 1+(240)(0.05)^{2}$

$\mathrm{n}=240 / 1+(240)(0.0025)$

$\mathrm{n}=240 / 1+0.6$

$\mathrm{n}=240 / 1.6$

$\mathrm{n}=150$

hence total 150 nurses were under consideration in this research.

\section{Ethical consideration}

In this research ethical consideration was preferred. For this purpose the permission was obtained from the ethical committee of the health care institution, before data collection. Permission will acquire a written approval from head of department of Lahore school of nursing in the form of consent. Furthermore inform written and verbal consent was taken before data collection from participants. Students were given with the right of autonomy and the nature and purpose of the study will informed prior to the implementation of any action. The risk related to this study was discussed before. Participants will have right to leave the study participation at any time. In this case other participants were added for the accomplishment of data information. Participants were informed about the aims of the study and secrecy of the collected data was assured. A written consent was taken from respondent those who were willing to participate in this study. All respondent were informed that their participation is highly appreciated and they can participate voluntarily. Participants were taken in confidence that all the collected information and records will remain confidential.

\section{Results and data analysis}

Results and data analysis was taken up through systematically and logically techniques after the accomplishment of data collection process.

\section{Budgets}

Expression of a financial plan for future expenses.

\section{RESULTS}

\section{Demographic Data}

Variables

Age
$>25$ years

35 years
Frequency (Percentage)

46(30.7\%)

$47(31.3 \%)$ 
International Journal of Health, Medicine and Nursing Practice ISSN 2710-1150 (Online)

Vol. 3, Issue No. 2, pp 61-106, 2021

$<45$ years

Total

Sex

Female

Male

Total

Qualification

Diploma degree

Associate degree

Bachelor degree

Total
$57(38 \%)$

$150(100 \%)$

$84(56 \%)$

66(44\%)

$150(100 \%)$

$120(80 \%)$

21(14\%)

$9(6 \%)$

$150(100 \%)$

\begin{tabular}{|c|c|c|}
\hline \multirow[t]{4}{*}{ Work duration (years) } & 8 & $123(82 \%)$ \\
\hline & 18 & $12(8 \%)$ \\
\hline & $>28$ & $15(10 \%)$ \\
\hline & Total & $150(100 \%)$ \\
\hline \multirow[t]{3}{*}{ Endoscopy Experience } & $<10$ years & $109(72.7 \%)$ \\
\hline & $>/=10$ years & $41(27.3 \%)$ \\
\hline & Total & $150(100 \%)$ \\
\hline \multirow[t]{3}{*}{ Training } & Yes & $121(80.7 \%)$ \\
\hline & No & $29(19.3 \%)$ \\
\hline & Total & $150(100 \%)$ \\
\hline \multirow[t]{3}{*}{ Vaccination (HBV) } & Yes & $123(82 \%)$ \\
\hline & No & $12(8 \%)$ \\
\hline & Total & $150(100 \%)$ \\
\hline
\end{tabular}

Tables and Figures: 
International Journal of Health, Medicine and Nursing Practice

ISSN 2710-1150 (Online)

Vol. 3, Issue No. 2, pp 61-106, 2021

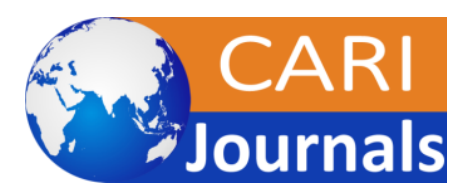

www.carijournals.org

\begin{tabular}{|l|r|r|r|r|}
\hline Age & Frequency & Percent & $\begin{array}{c}\text { Valid } \\
\text { Percent }\end{array}$ & $\begin{array}{c}\text { Cumulative } \\
\text { Percent }\end{array}$ \\
\hline$>25$ & 46 & $30.7 \%$ & $30.7 \%$ & $30.7 \%$ \\
\hline 35 & 47 & $31.3 \%$ & $31.3 \%$ & $62 \%$ \\
\hline$<45$ & 57 & $38 \%$ & $38 \%$ & $100 \%$ \\
\hline Total & 150 & $100 \%$ & $100 \%$ & \\
\hline
\end{tabular}

Table 1

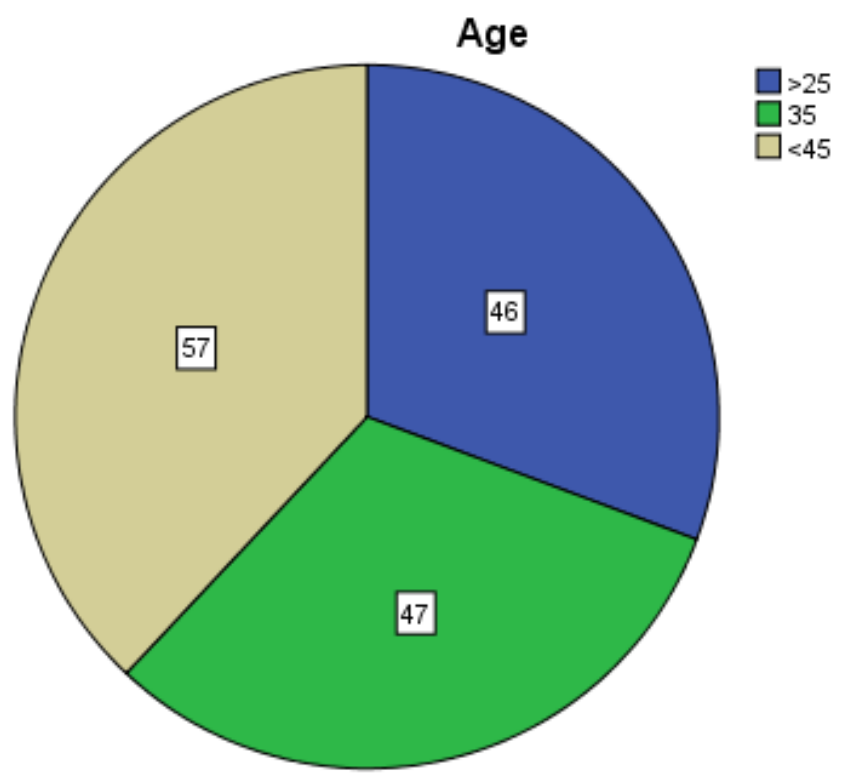

FIG 1

Figure 1 show that participants were more than 45 years old (38\%) while many of them were 35 years old $(31.3 \%)$ and some of them were less than 25 years old $(30.7 \%)$.

\begin{tabular}{|l|r|r|r|r|}
\hline Sex & Frequency & Percent & \multicolumn{1}{c|}{$\begin{array}{c}\text { Valid } \\
\text { Percent }\end{array}$} & $\begin{array}{c}\text { Cumulative } \\
\text { Percent }\end{array}$ \\
\hline Female & 84 & $56 \%$ & $56 \%$ & $56 \%$ \\
\hline Male & 66 & $44 \%$ & $44 \%$ & $100 \%$ \\
\hline
\end{tabular}


International Journal of Health, Medicine and Nursing Practice

ISSN 2710-1150 (Online)

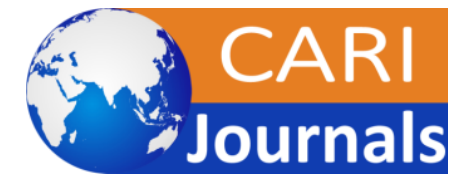

Vol. 3, Issue No. 2, pp 61-106, 2021

www.carijournals.org

\begin{tabular}{|l|r|r|r|l|}
\hline Total & 150 & $100 \%$ & $100 \%$ & \\
\hline
\end{tabular}

Table 2

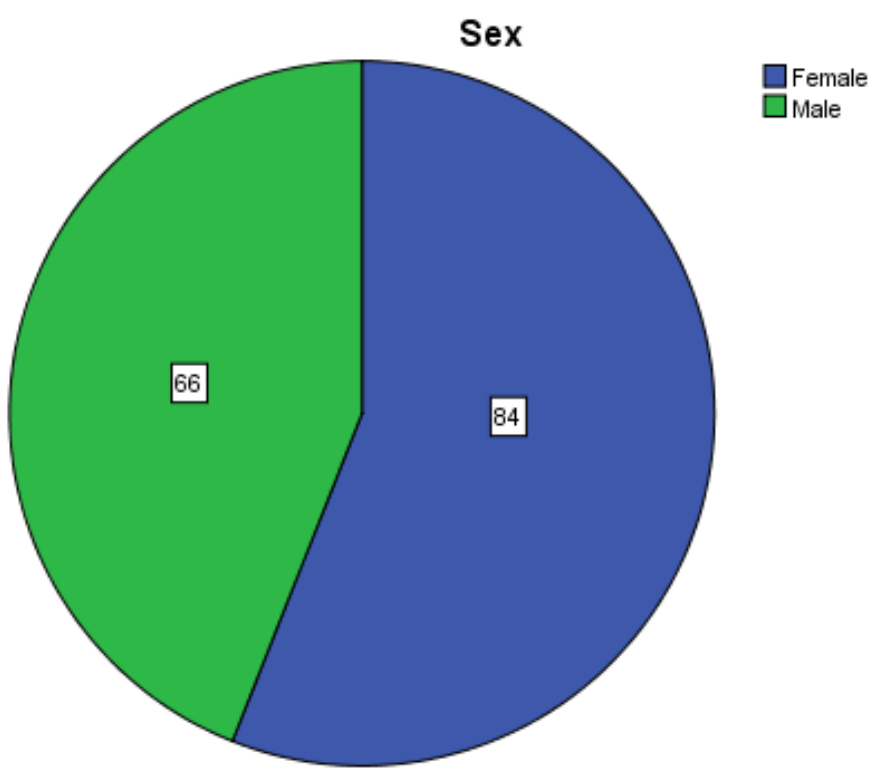

Figure 2 shows that participants were more females (56\%) than males $(44 \%)$.

\begin{tabular}{|l|r|r|r|r|}
\hline Qualification & Frequency & Percent & $\begin{array}{c}\text { Valid } \\
\text { Percent }\end{array}$ & $\begin{array}{c}\text { Cumulative } \\
\text { Percent }\end{array}$ \\
\hline $\begin{array}{l}\text { Diploma } \\
\text { Degree }\end{array}$ & 120 & $80 \%$ & $80 \%$ & $80 \%$ \\
\hline $\begin{array}{l}\text { Associate } \\
\text { Degree }\end{array}$ & 21 & $14 \%$ & $14 \%$ & $94 \%$ \\
\hline $\begin{array}{l}\text { Bachelor } \\
\text { Degree }\end{array}$ & 9 & $6 \%$ & $6 \%$ & $100 \%$ \\
\hline Total & 150 & $100 \%$ & $100 \%$ & \\
\hline
\end{tabular}

Table 3 
International Journal of Health, Medicine and Nursing Practice ISSN 2710-1150 (Online)

Vol. 3, Issue No. 2, pp 61-106, 2021

www.carijournals.org

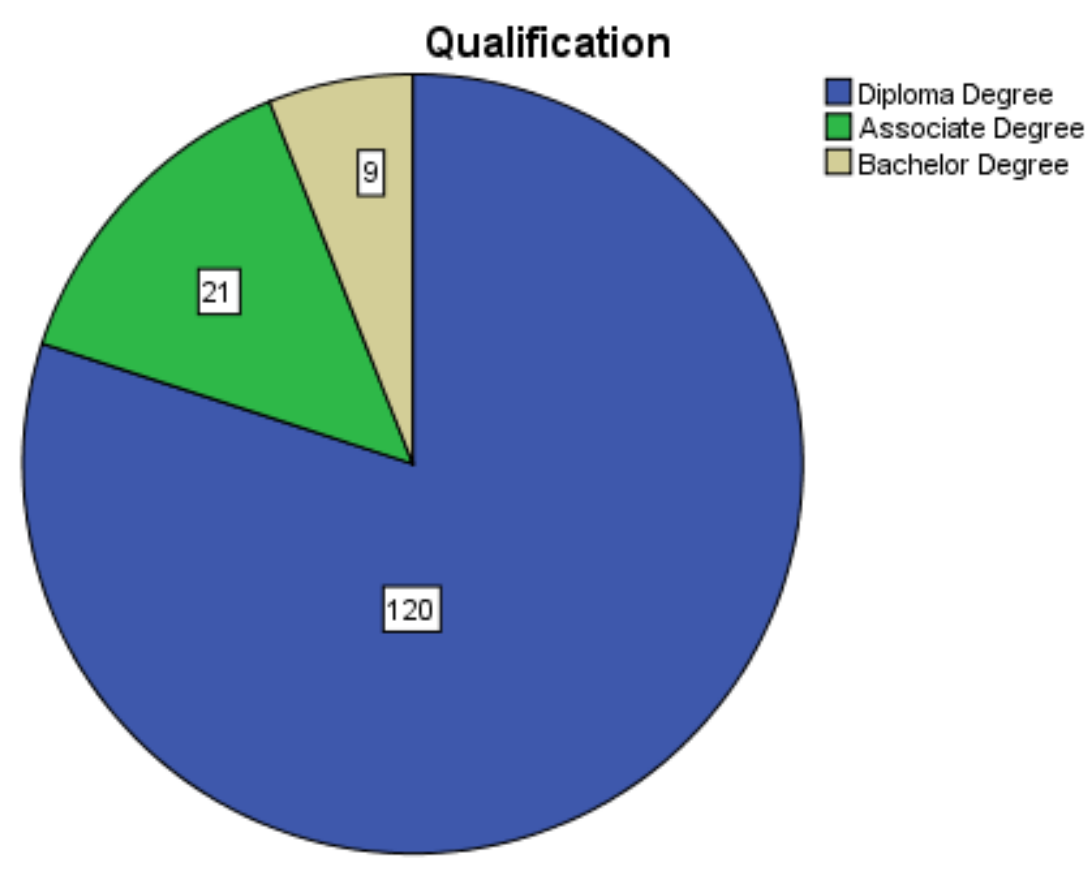

FIG 3

Figure 3 shows that participants were more qualified in diploma degree $(80 \%)$ while some of them have associate degree (14\%) but very few are graduated in bachelors degree $(6 \%)$.

\begin{tabular}{|l|r|r|r|r|}
\hline $\begin{array}{l}\text { Work } \\
\text { Duration } \\
\text { (years) }\end{array}$ & Frequency & \multicolumn{1}{|c|}{ Percent } & \multicolumn{1}{|c|}{$\begin{array}{c}\text { Valid } \\
\text { Percent }\end{array}$} & $\begin{array}{c}\text { Cumulative } \\
\text { Percent }\end{array}$ \\
\hline 8 & 123 & $82 \%$ & $82 \%$ & $82 \%$ \\
\hline 18 & 12 & $8 \%$ & $8 \%$ & $90 \%$ \\
\hline$>28$ & 15 & $10 \%$ & $10 \%$ & $100 \%$ \\
\hline Total & 150 & $100 \%$ & $100 \%$ & \\
\hline
\end{tabular}

Table 4 
International Journal of Health, Medicine and Nursing Practice ISSN 2710-1150 (Online)

Vol. 3, Issue No. 2, pp 61-106, 2021

www.carijournals.org

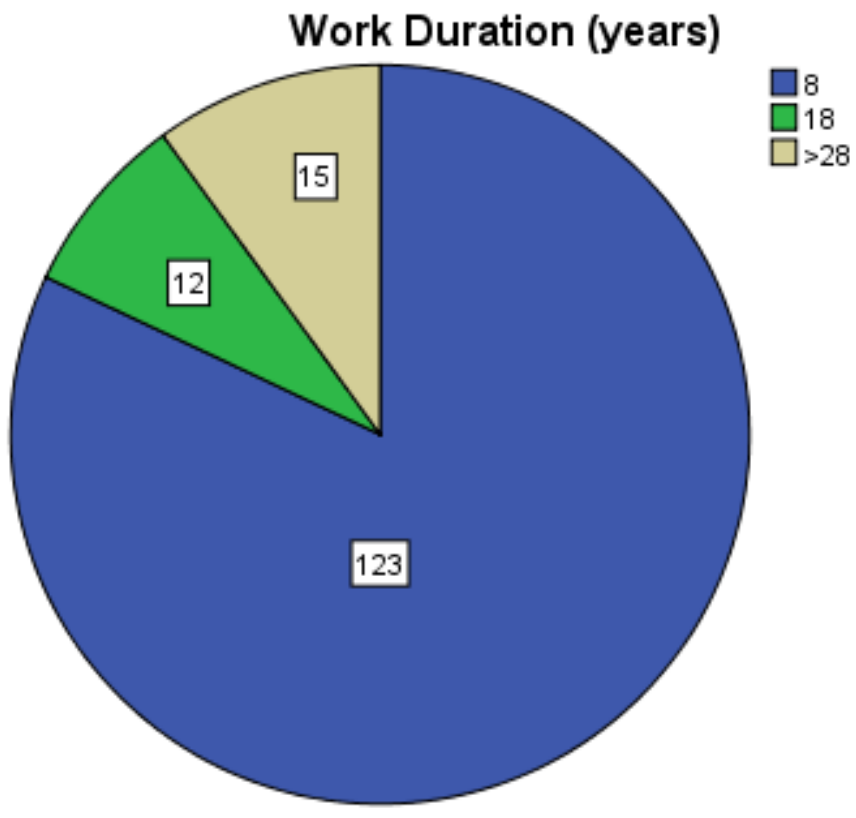

FIG 4

Figure 4 shows that the work duration of participants is mostly 8 years $(82 \%)$, while some have $>28$ years duration $(\mathbf{1 0 \%})$ but few of them have 18 years of work duration $(8 \%)$.

\begin{tabular}{|l|r|r|r|r|}
\hline $\begin{array}{l}\text { Endoscopy } \\
\text { Experience }\end{array}$ & Frequency & Percent & $\begin{array}{c}\text { Valid } \\
\text { Percent }\end{array}$ & $\begin{array}{c}\text { Cumulative } \\
\text { Percent }\end{array}$ \\
\hline$<10$ years & 109 & $72.7 \%$ & $72.7 \%$ & $72.7 \%$ \\
\hline$>10$ years & 41 & $27.3 \%$ & $27.3 \%$ & $100 \%$ \\
\hline Total & 150 & $100 \%$ & $100 \%$ & \\
\hline
\end{tabular}

Table 5 
International Journal of Health, Medicine and Nursing Practice

ISSN 2710-1150 (Online)

Vol. 3, Issue No. 2, pp 61-106, 2021

www.carijournals.org

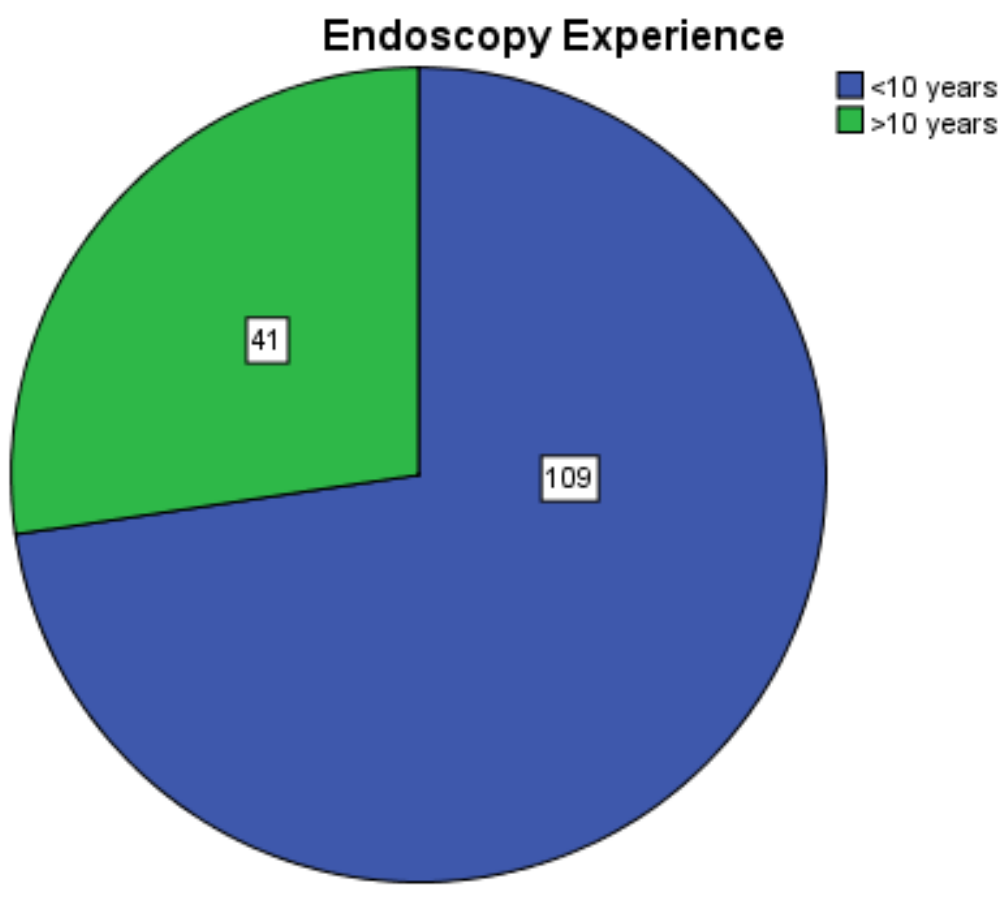

FIG 5

Figure 5 shows that endoscopy experience of participants is mostly less than 10 years $\mathbf{7 2 . 7 \%}$ ) while some of them have experience greater than ten years $(\mathbf{2 7 . 3 \%})$.

\begin{tabular}{|l|r|r|r|r|}
\hline Training & Frequency & \multicolumn{1}{|c|}{ Percent } & $\begin{array}{c}\text { Valid } \\
\text { Percent }\end{array}$ & $\begin{array}{c}\text { Cumulative } \\
\text { Percent }\end{array}$ \\
\hline Yes & 121 & $80.7 \%$ & $80.7 \%$ & $80.7 \%$ \\
\hline No & 29 & $19.3 \%$ & $19.3 \%$ & $100 \%$ \\
\hline Total & 150 & $100 \%$ & $100 \%$ & \\
\hline
\end{tabular}

Table 6 
International Journal of Health, Medicine and Nursing Practice ISSN 2710-1150 (Online)

Vol. 3, Issue No. 2, pp 61-106, 2021

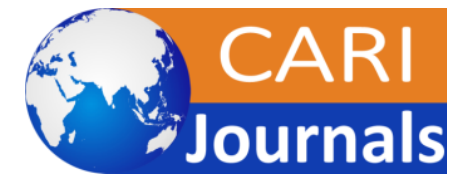

www.carijournals.org

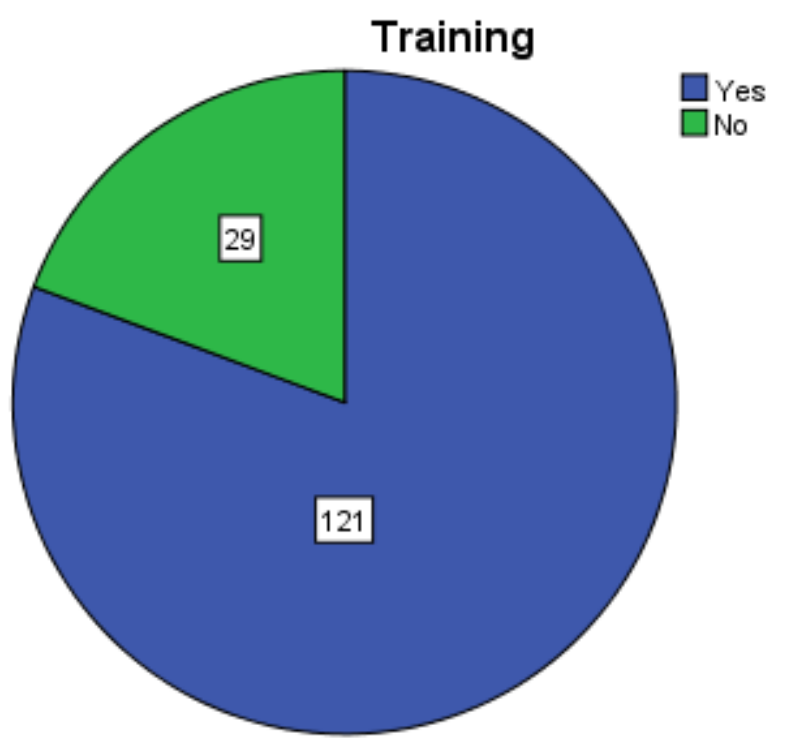

FIG 6

Figure 6 shows that large percentage of participants have received training for endoscopy procedures $(\mathbf{8 0 . 7 \%})$ while many of them disagreed to this statement $(19.3 \%)$.

\begin{tabular}{|l|r|r|r|r|}
\hline $\begin{array}{l}\text { Vaccination } \\
\text { (HBV) }\end{array}$ & Frequency & Percent & $\begin{array}{c}\text { Valid } \\
\text { Percent }\end{array}$ & $\begin{array}{c}\text { Cumulative } \\
\text { Percent }\end{array}$ \\
\hline Yes & 150 & $100 \%$ & $100 \%$ & $100 \%$ \\
\hline Total & 150 & $100 \%$ & $100 \%$ & \\
\hline
\end{tabular}

Table 7 
International Journal of Health, Medicine and Nursing Practice ISSN 2710-1150 (Online)

Vol. 3, Issue No. 2, pp 61-106, 2021

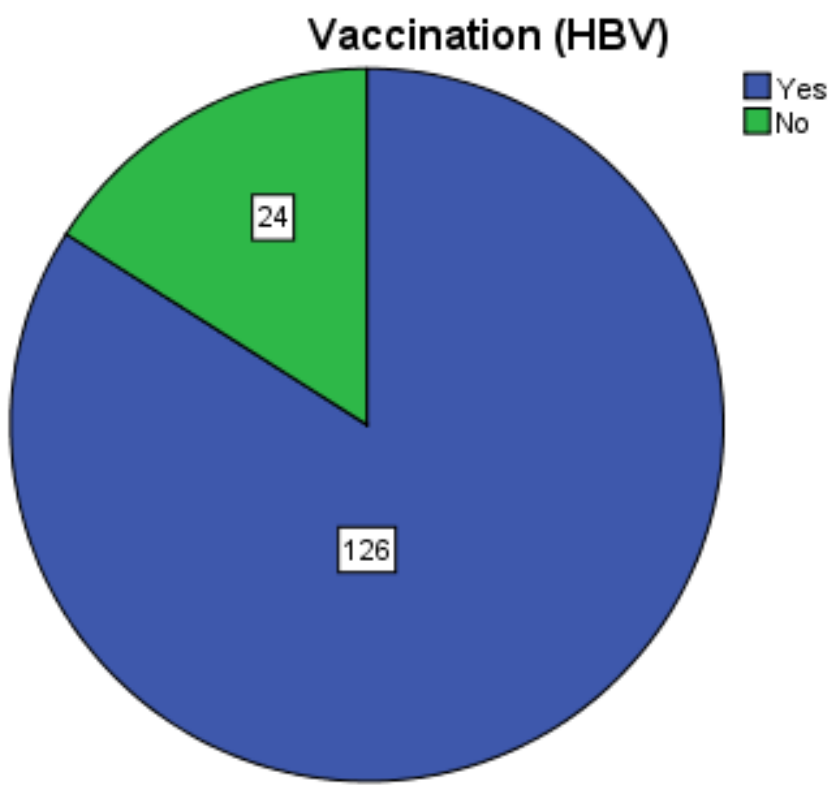

FIG 7

Figure 7 shows that all of participants have received vaccination of HBV (100\%).

\begin{tabular}{|l|r|r|r|r|}
\hline & Frequency & Percent & $\begin{array}{c}\text { Valid } \\
\text { Percent }\end{array}$ & $\begin{array}{c}\text { Cumulative } \\
\text { Percent }\end{array}$ \\
\hline Yes & 150 & $100 \%$ & $100 \%$ & $100 \%$ \\
\hline Total & 150 & $100 \%$ & $100 \%$ & \\
\hline
\end{tabular}

Table 8 
International Journal of Health, Medicine and Nursing Practice ISSN 2710-1150 (Online)

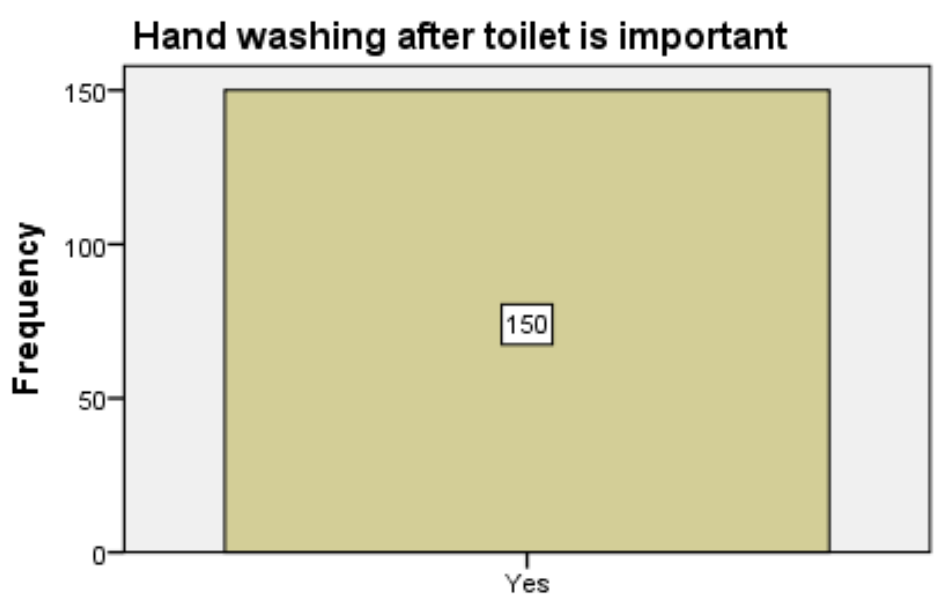

FIG 8

Figure 8 shows that all participants agreed to it that hand washing after using toilet is necessary $(100 \%)$.

\begin{tabular}{|l|r|r|r|r|}
\hline & Frequency & Percent & $\begin{array}{c}\text { Valid } \\
\text { Percent }\end{array}$ & $\begin{array}{c}\text { Cumulative } \\
\text { Percent }\end{array}$ \\
\hline Yes & 150 & $100 \%$ & $100 \%$ & $100 \%$ \\
\hline Total & 150 & $100 \%$ & $100 \%$ & \\
\hline
\end{tabular}

Table 9 
International Journal of Health, Medicine and Nursing Practice ISSN 2710-1150 (Online)

Vol. 3, Issue No. 2, pp 61-106, 2021

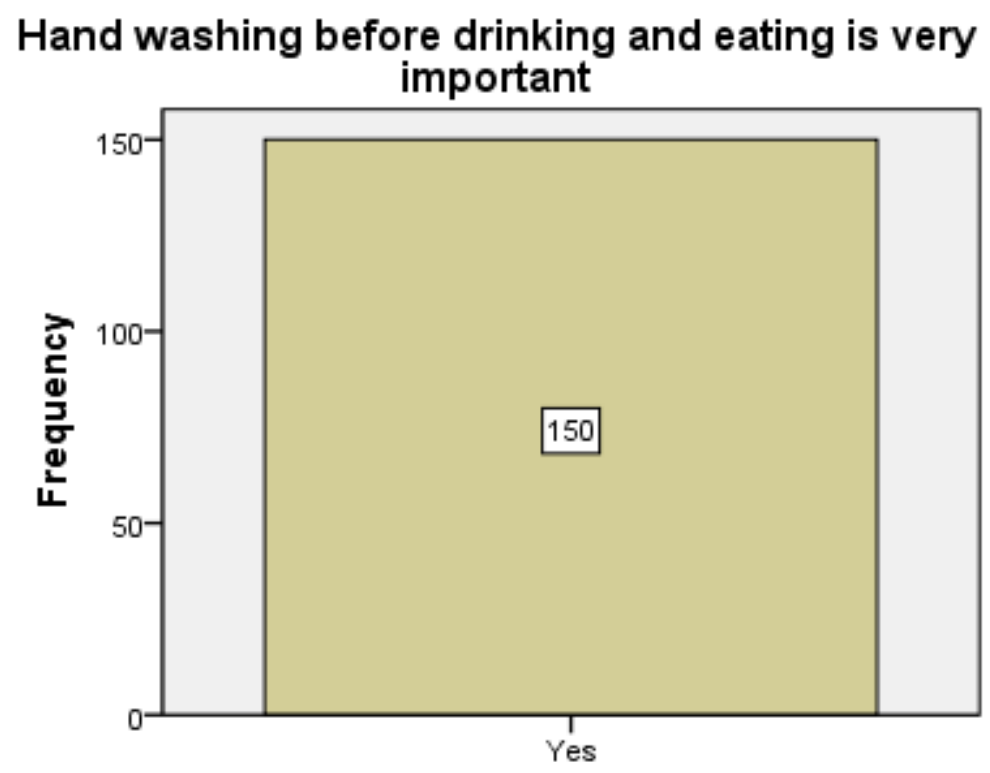

FIG 9

Figure 9 shows that all participants agreed to it that hand washing before drinking and eating is important $(100 \%)$.

\begin{tabular}{|l|r|r|r|r|}
\hline & Frequency & \multicolumn{1}{|c|}{ Percent } & $\begin{array}{c}\text { Valid } \\
\text { Percent }\end{array}$ & $\begin{array}{c}\text { Cumulative } \\
\text { Percent }\end{array}$ \\
\hline Yes & 133 & $88.7 \%$ & $88.7 \%$ & $88.7 \%$ \\
\hline No & 4 & $2.7 \%$ & $2.7 \%$ & $91.3 \%$ \\
\hline $\begin{array}{l}\text { Don't } \\
\text { know }\end{array}$ & 13 & $8.7 \%$ & $8.7 \%$ & $100 \%$ \\
\hline Total & 150 & $100 \%$ & $100 \%$ & \\
\hline
\end{tabular}


International Journal of Health, Medicine and Nursing Practice ISSN 2710-1150 (Online)

Vol. 3, Issue No. 2, pp 61-106, 2021

Table 10

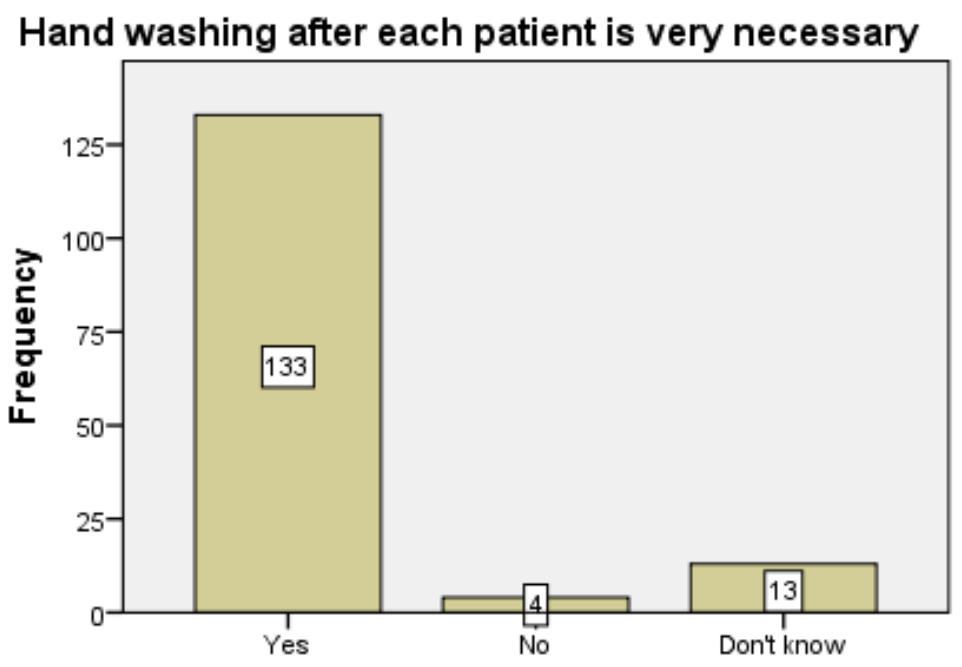

FIG 10

Figure 10 shows that all participants agreed to it that hand washing after each patient care is necessary $(\mathbf{1 0 0 \%})$.

Table 11

\begin{tabular}{|l|r|r|r|r|}
\hline & Frequency & Percent & $\begin{array}{c}\text { Valid } \\
\text { Percent }\end{array}$ & $\begin{array}{c}\text { Cumulative } \\
\text { Percent }\end{array}$ \\
\hline Yes & 150 & $100 \%$ & $100 \%$ & $100 \%$ \\
\hline Total & 150 & $100 \%$ & $100 \%$ & \\
\hline
\end{tabular}


International Journal of Health, Medicine and Nursing Practice ISSN 2710-1150 (Online)

Vol. 3, Issue No. 2, pp 61-106, 2021

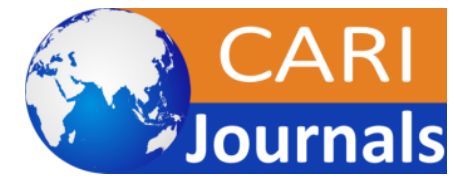

$\underline{\text { www.carijournals.org }}$

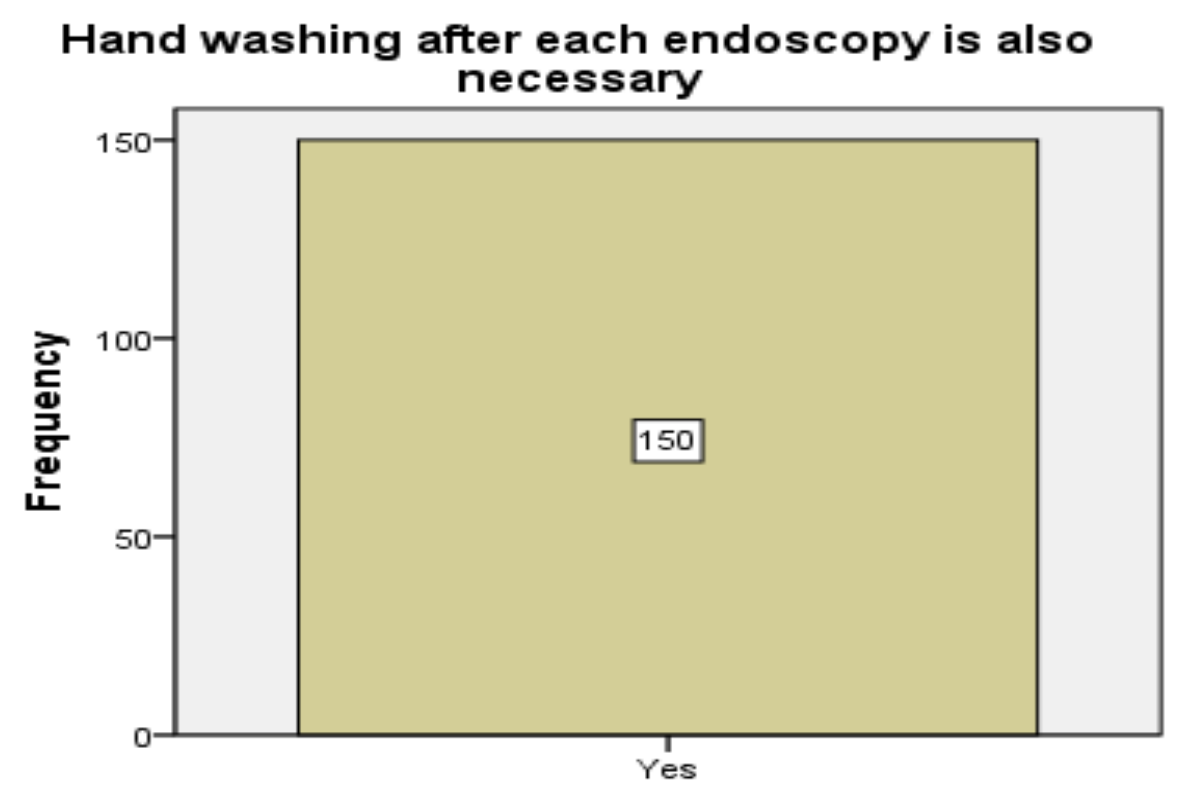

FIG 11

Figure 11 shows that all participants agreed to it that hand washing after each endoscopy is necessary $(100 \%)$.

Table 12

\begin{tabular}{|l|r|r|r|r|}
\hline & Frequency & \multicolumn{1}{|c|}{ Percent } & $\begin{array}{c}\text { Valid } \\
\text { Percent }\end{array}$ & $\begin{array}{c}\text { Cumulative } \\
\text { Percent }\end{array}$ \\
\hline Yes & 131 & $87.3 \%$ & $87.3 \%$ & $87.3 \%$ \\
\hline No & 2 & $1.3 \%$ & $1.3 \%$ & $88.7 \%$ \\
\hline $\begin{array}{l}\text { Don't } \\
\text { know }\end{array}$ & 17 & $11.3 \%$ & $11.3 \%$ & $100 \%$ \\
\hline Total & 150 & $100 \%$ & $100 \%$ & \\
\hline
\end{tabular}


International Journal of Health, Medicine and Nursing Practice

ISSN 2710-1150 (Online)

Vol. 3, Issue No. 2, pp 61-106, 2021

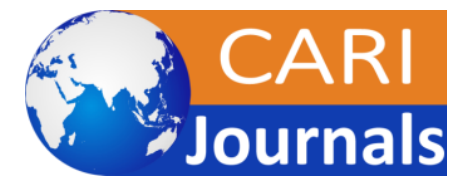

$\underline{\text { www.carijournals.org }}$

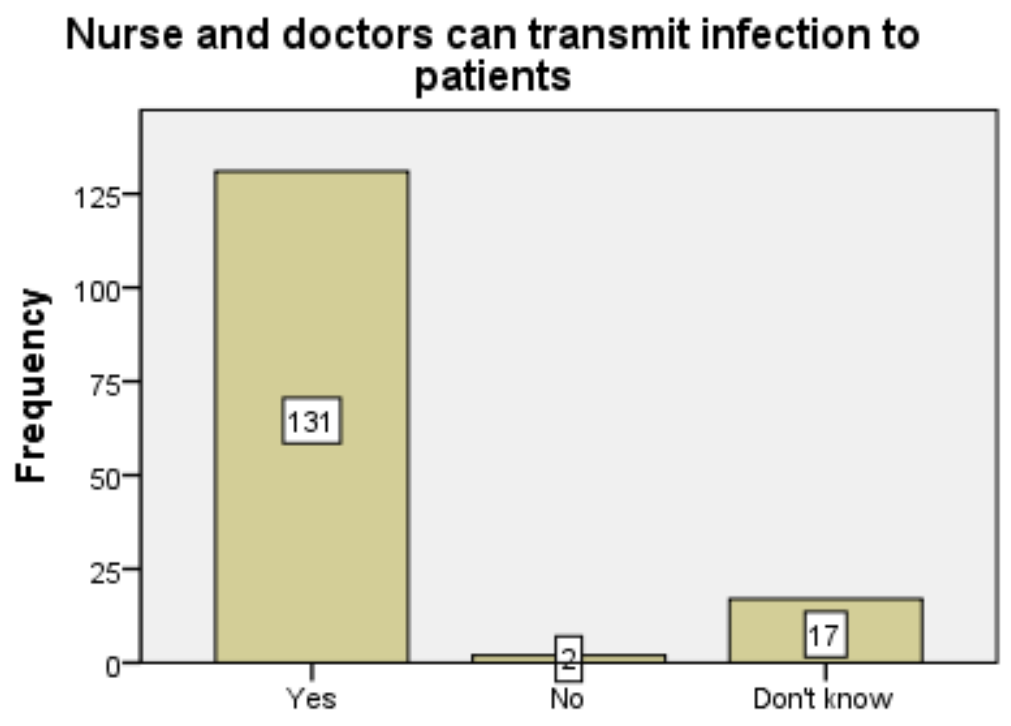

FIG 12

Figure 12 shows that many participants agreed to it that nurses and doctors can transmit infections to the patients $(87.3 \%)$ some answered that they don't know (11.3\%) but few said no to it $(1.3 \%)$

Table 13

\begin{tabular}{|l|r|r|r|r|}
\hline & Frequency & \multicolumn{1}{|c|}{ Percent } & \multicolumn{1}{c|}{$\begin{array}{c}\text { Valid } \\
\text { Percent }\end{array}$} & $\begin{array}{c}\text { Cumulative } \\
\text { Percent }\end{array}$ \\
\hline Yes & 124 & $82.7 \%$ & $82.7 \%$ & $82.7 \%$ \\
\hline No & 1 & $.7 \%$ & $.7 \%$ & $83.3 \%$ \\
\hline $\begin{array}{l}\text { Don't } \\
\text { know }\end{array}$ & 25 & $16.7 \%$ & $16.7 \%$ & $100 \%$ \\
\hline Total & 150 & $100 \%$ & $100 \%$ & \\
\hline
\end{tabular}


International Journal of Health, Medicine and Nursing Practice ISSN 2710-1150 (Online)

Vol. 3, Issue No. 2, pp 61-106, 2021

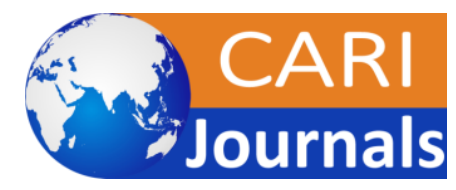

www.carijournals.org

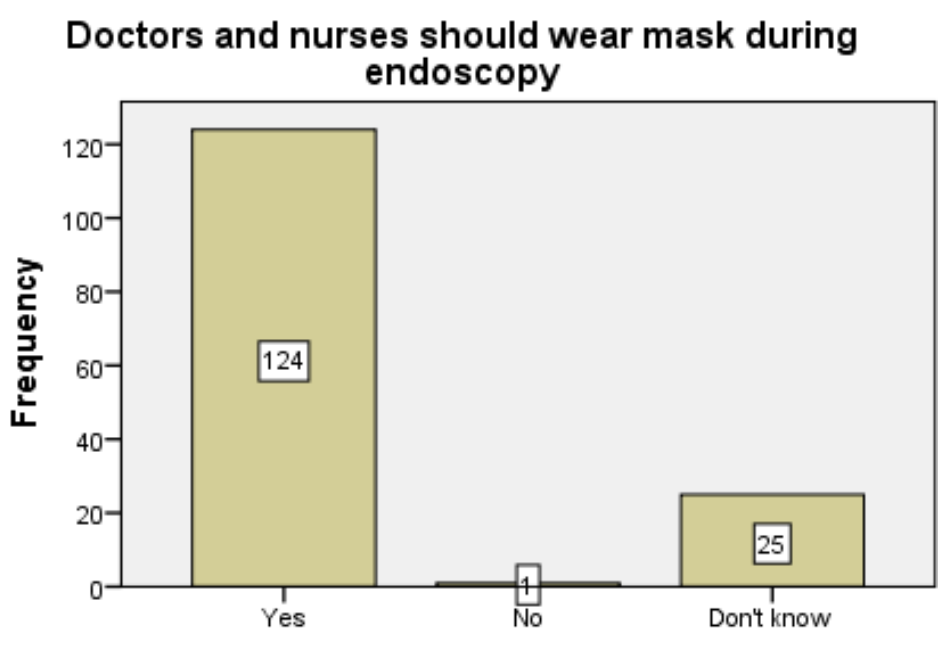

FIG 13

Figure 13 shows that many participants agreed to it that nurses and doctors should wear masks during endoscopy $(82.7 \%)$ some answered that they don't know $(16.7 \%)$ but few said no to it $(0.7 \%)$

Table 14

\begin{tabular}{|l|r|r|r|r|}
\hline & Frequency & \multicolumn{1}{|c|}{ Percent } & \multicolumn{1}{c|}{$\begin{array}{c}\text { Valid } \\
\text { Percent }\end{array}$} & $\begin{array}{c}\text { Cumulative } \\
\text { Percent }\end{array}$ \\
\hline Yes & 131 & $87.3 \%$ & $87.3 \%$ & $87.3 \%$ \\
\hline $\begin{array}{l}\text { Don't } \\
\text { know }\end{array}$ & 19 & $12.7 \%$ & $12.7 \%$ & $100 \%$ \\
\hline Total & 150 & $100 \%$ & $100 \%$ & \\
\hline
\end{tabular}


International Journal of Health, Medicine and Nursing Practice ISSN 2710-1150 (Online)

Vol. 3, Issue No. 2, pp 61-106, 2021

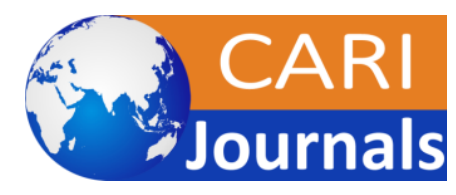

www.carijournals.org

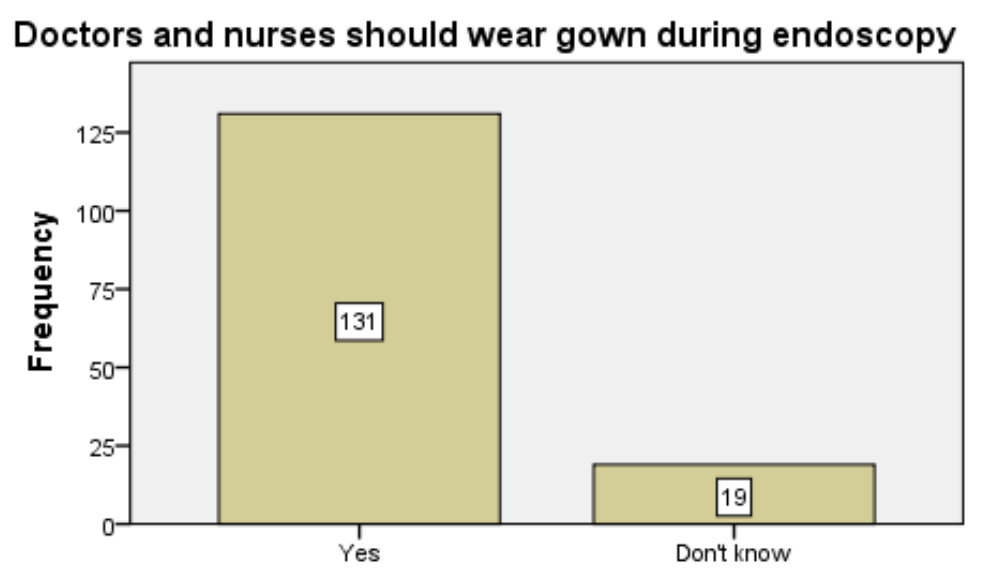

FIG 14

Figure 14 shows that many participants agreed to it that nurses and doctors should wear gowns during endoscopy $(87.3 \%)$ some answered that they don't know $(12.7 \%)$.

\begin{tabular}{|l|r|r|r|r|}
\hline & Frequency & \multicolumn{1}{|c|}{ Percent } & $\begin{array}{c}\text { Valid } \\
\text { Percent }\end{array}$ & $\begin{array}{c}\text { Cumulative } \\
\text { Percent }\end{array}$ \\
\hline Yes & 129 & $86 \%$ & $86 \%$ & $86 \%$ \\
\hline $\begin{array}{l}\text { Don't } \\
\text { know }\end{array}$ & 21 & $14 \%$ & $14 \%$ & $100 \%$ \\
\hline Total & 150 & $100 \%$ & $100 \%$ & \\
\hline
\end{tabular}

Table 15 
International Journal of Health, Medicine and Nursing Practice ISSN 2710-1150 (Online)

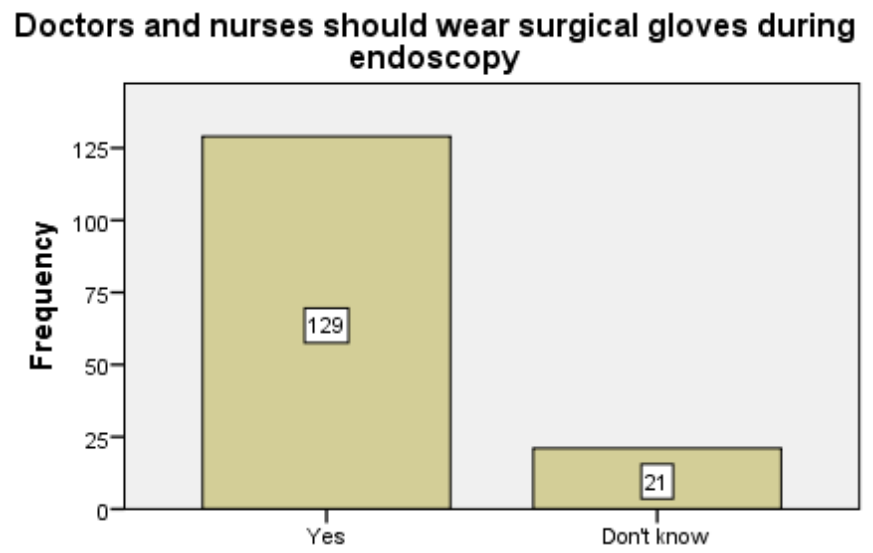

FIG 15

Figure 15 shows that many participants agreed to it that nurses and doctors should wear surgical gloves during endoscopy (86\%) some answered that they don't know (14\%).

\begin{tabular}{|l|r|r|r|r|}
\hline & Frequency & \multicolumn{1}{|c|}{ Percent } & \multicolumn{1}{c|}{$\begin{array}{c}\text { Valid } \\
\text { Percent }\end{array}$} & $\begin{array}{c}\text { Cumulative } \\
\text { Percent }\end{array}$ \\
\hline Yes & 144 & $96 \%$ & $96 \%$ & $96 \%$ \\
\hline $\begin{array}{l}\text { Don't } \\
\text { know }\end{array}$ & 6 & $4 \%$ & $4 \%$ & $100 \%$ \\
\hline Total & 150 & $100 \%$ & $100 \%$ & \\
\hline
\end{tabular}

Table 16 
International Journal of Health, Medicine and Nursing Practice ISSN 2710-1150 (Online)

Vol. 3, Issue No. 2, pp 61-106, 2021

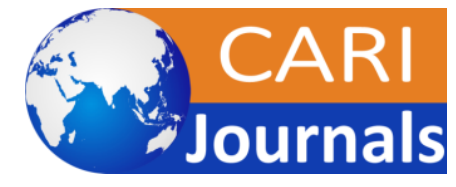

$\underline{\text { www.carijournals.org }}$

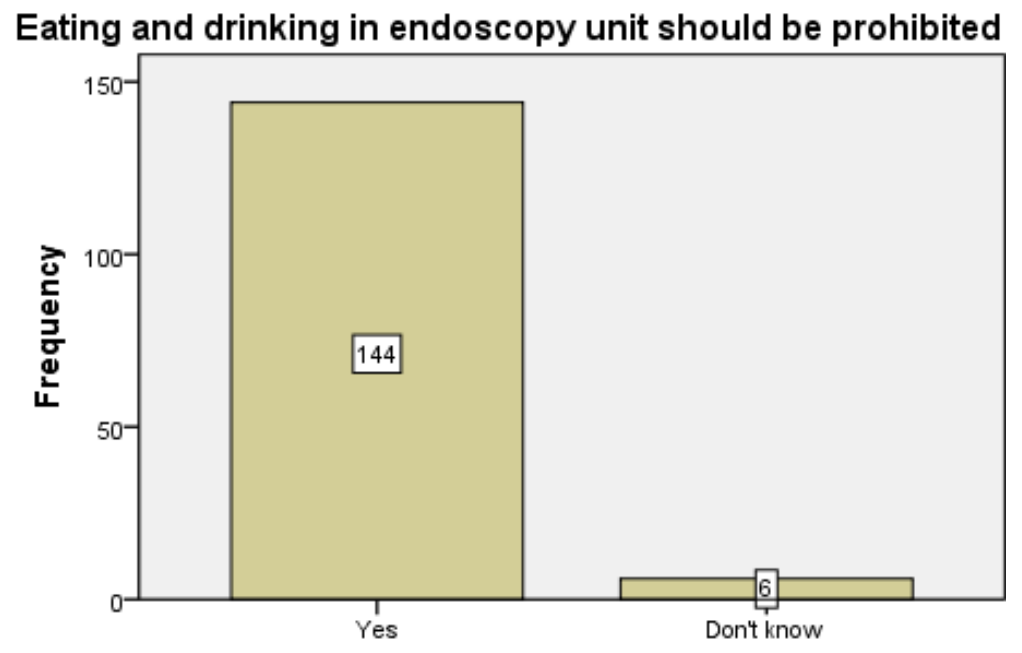

Figure 16 shows that many participants agreed to it that eating and drinking in endoscopy unit should be prohibited (96\%) some answered that they don't know $(4 \%)$.

\begin{tabular}{|l|r|r|r|r|}
\hline & Frequency & Percent & $\begin{array}{c}\text { Valid } \\
\text { Percent }\end{array}$ & $\begin{array}{c}\text { Cumulative } \\
\text { Percent }\end{array}$ \\
\hline Yes & 150 & 100.0 & 100.0 & 100.0 \\
\hline Total & 150 & $100 \%$ & $100 \%$ & \\
\hline
\end{tabular}

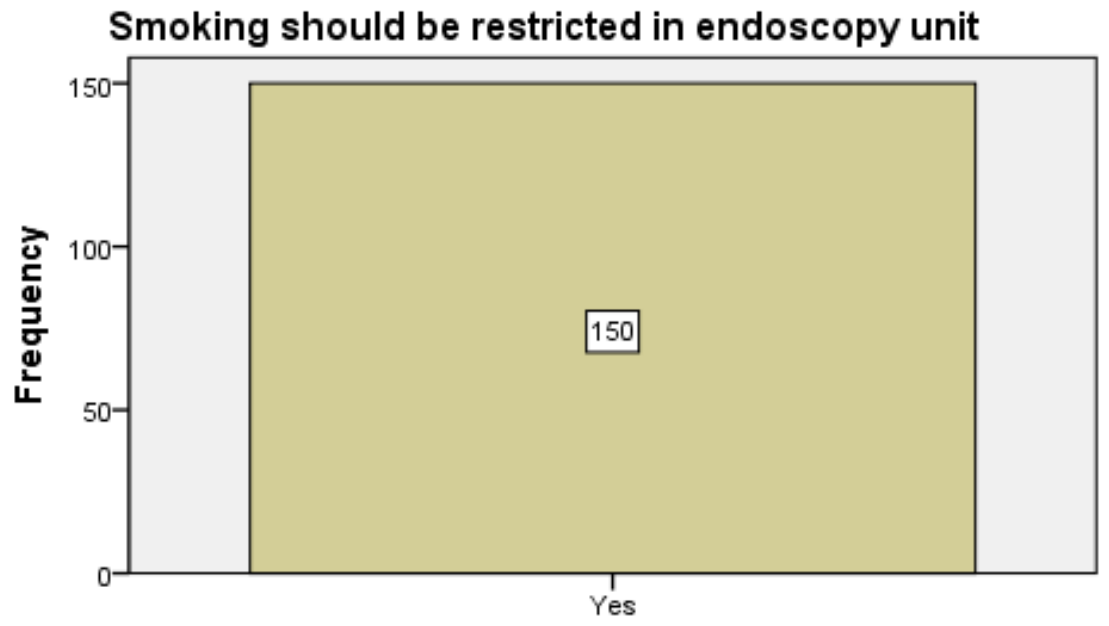


International Journal of Health, Medicine and Nursing Practice ISSN 2710-1150 (Online)

Vol. 3, Issue No. 2, pp 61-106, 2021

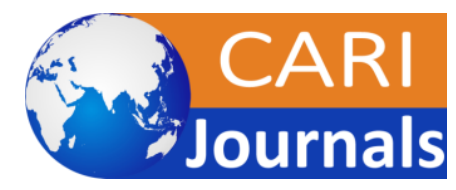

www.carijournals.org

Figure 17 shows that all participants agreed to it that smoking in endoscopy unit should be strictly restricted $(\mathbf{1 0 0 \%})$.

\begin{tabular}{|l|r|r|r|r|}
\hline & Frequency & \multicolumn{1}{|c|}{ Percent } & $\begin{array}{c}\text { Valid } \\
\text { Percent }\end{array}$ & \multicolumn{1}{c|}{$\begin{array}{c}\text { Cumulative } \\
\text { Percent }\end{array}$} \\
\hline Agree & 84 & $56 \%$ & $56 \%$ & $56 \%$ \\
\hline Sometimes & 66 & $44 \%$ & $44 \%$ & $100 \%$ \\
\hline Total & 150 & $100 \%$ & $100 \%$ & \\
\hline
\end{tabular}

Table 18

Importance of providing comfortable place for patients

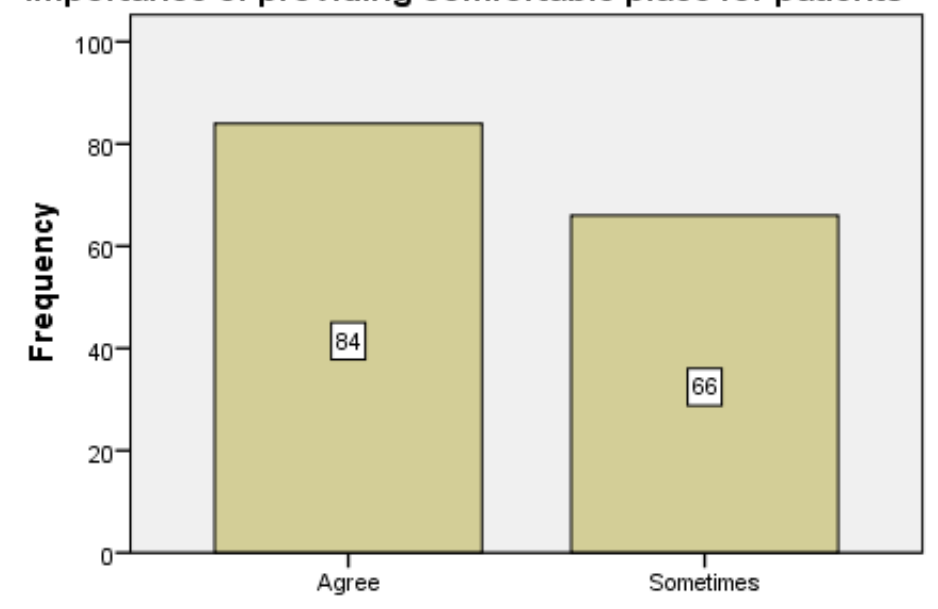

FIG 18

Figure 18 shows that many participants agreed to it that it is very important to provide comfortable place to patient $(56 \%)$ some answered that sometimes its important $(44 \%)$.

\begin{tabular}{|l|l|l|l|l|}
\hline & Frequency & Percent & $\begin{array}{c}\text { Valid } \\
\text { Percent }\end{array}$ & $\begin{array}{c}\text { Cumulative } \\
\text { Percent }\end{array}$ \\
\hline
\end{tabular}


International Journal of Health, Medicine and Nursing Practice

ISSN 2710-1150 (Online)

Vol. 3, Issue No. 2, pp 61-106, 2021

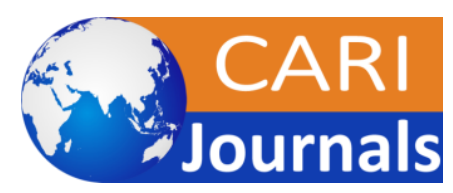

www.carijournals.org

\begin{tabular}{|l|r|r|r|r|}
\hline Agree & 53 & $35.3 \%$ & $35.3 \%$ & $35.3 \%$ \\
\hline Sometimes & 35 & $23.3 \%$ & $23.3 \%$ & $58.7 \%$ \\
\hline Disagree & 62 & $41.3 \%$ & $41.3 \%$ & $100 \%$ \\
\hline Total & 150 & $100 \%$ & $100 \%$ & \\
\hline
\end{tabular}

Table 19

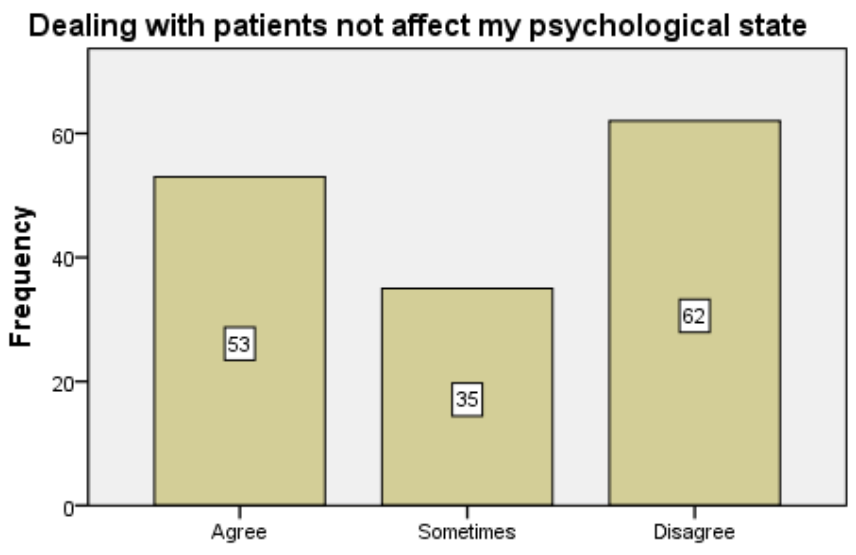

FIG 19

Figure 19 shows that many participants disagreed to it that patient dealing do not effects psychological state $(41.3 \%)$ many of them agreed to it $(35.3 \%)$ while some answered sometimes $(23.3 \%)$.

\begin{tabular}{|l|r|r|r|r|}
\hline & Frequency & \multicolumn{1}{|c|}{ Percent } & $\begin{array}{c}\text { Valid } \\
\text { Percent }\end{array}$ & $\begin{array}{c}\text { Cumulative } \\
\text { Percent }\end{array}$ \\
\hline Agree & 21 & $14 \%$ & $14 \%$ & $14 \%$ \\
\hline Sometimes & 41 & $27.3 \%$ & $27.3 \%$ & $41.3 \%$ \\
\hline Disagree & 88 & $58.7 \%$ & $58.7 \%$ & $100 \%$ \\
\hline Total & 150 & $100 \%$ & $100 \%$ & \\
\hline
\end{tabular}


International Journal of Health, Medicine and Nursing Practice ISSN 2710-1150 (Online)

Vol. 3, Issue No. 2, pp 61-106, 2021

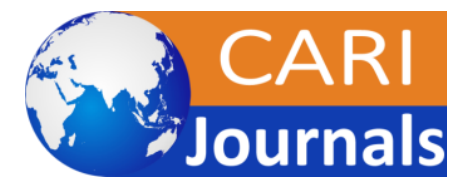

www.carijournals.org

Table 20

Fairness of endoscopic patient blocks delay providing

nursing care

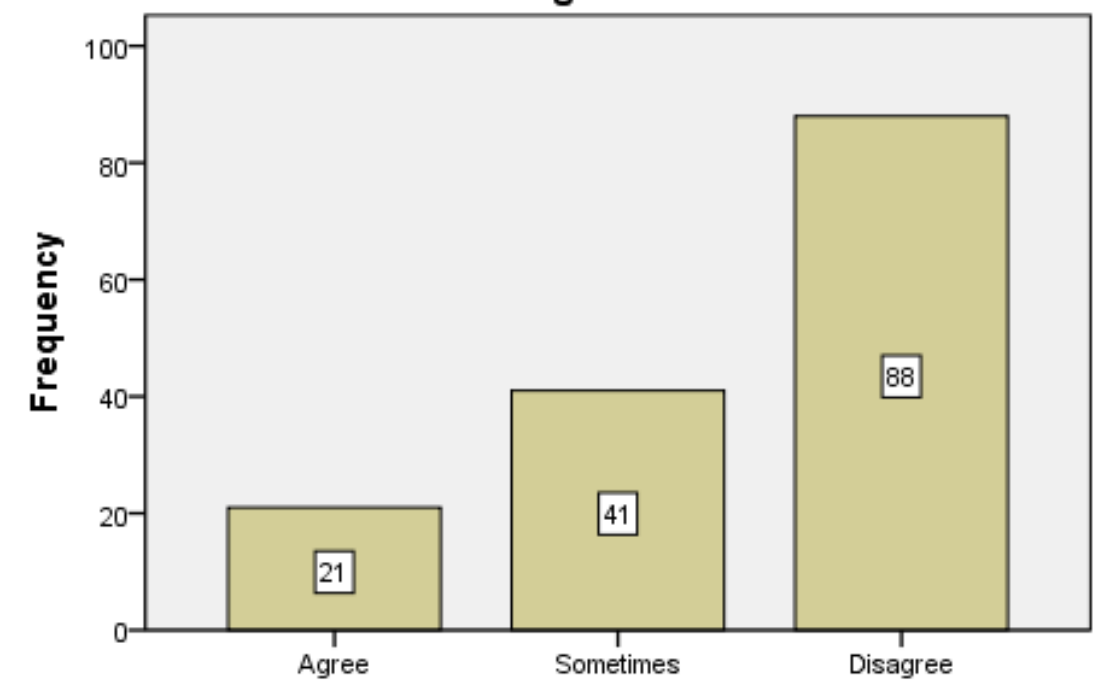

FIG 20

Figure 20 shows that many participants disagreed to it that fairness of endoscopic patient blocks delay in providing nursing care (58.7\%) many of them answered sometimes $(27.3 \%)$ while some agreed to it $(\mathbf{1 4 \%})$.

\begin{tabular}{|l|r|r|r|r|}
\hline & Frequency & \multicolumn{1}{|c|}{ Percent } & \multicolumn{1}{c|}{$\begin{array}{c}\text { Valid } \\
\text { Percent }\end{array}$} & $\begin{array}{c}\text { Cumulative } \\
\text { Percent }\end{array}$ \\
\hline Agree & 88 & $58.7 \%$ & $58.7 \%$ & $58.7 \%$ \\
\hline Sometimes & 51 & $34 \%$ & $34 \%$ & $92.7 \%$ \\
\hline Disagree & 11 & $7.3 \%$ & $7.3 \%$ & $100 \%$ \\
\hline Total & 150 & $100 \%$ & $100 \%$ & \\
\hline
\end{tabular}

Table 21 
International Journal of Health, Medicine and Nursing Practice ISSN 2710-1150 (Online)

Vol. 3, Issue No. 2, pp 61-106, 2021

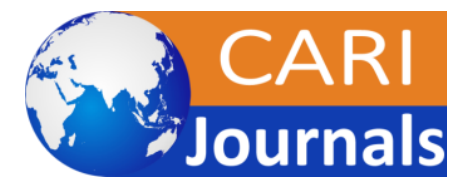

www.carijournals.org

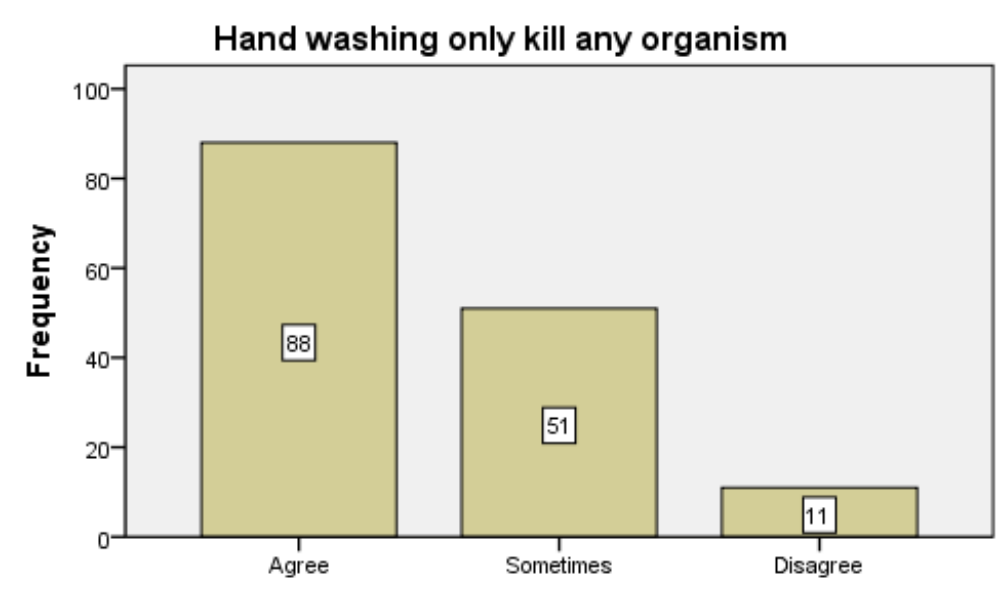

FIG 21

Figure 21 shows that many participants agreed to it that hand washing kills only organisms $(58.7 \%)$ many of them answered sometimes $(34 \%)$ while some disagreed to it $(7.3 \%)$.

\begin{tabular}{|l|r|r|r|r|}
\hline & Frequency & \multicolumn{1}{|c|}{ Percent } & $\begin{array}{c}\text { Valid } \\
\text { Percent }\end{array}$ & $\begin{array}{c}\text { Cumulative } \\
\text { Percent }\end{array}$ \\
\hline Agree & 120 & $80 \%$ & $80 \%$ & $80 \%$ \\
\hline Sometimes & 30 & $20 \%$ & $20 \%$ & $100 \%$ \\
\hline Total & 150 & $100 \%$ & $100 \%$ & \\
\hline
\end{tabular}

Table 22 
International Journal of Health, Medicine and Nursing Practice ISSN 2710-1150 (Online)

Vol. 3, Issue No. 2, pp 61-106, 2021

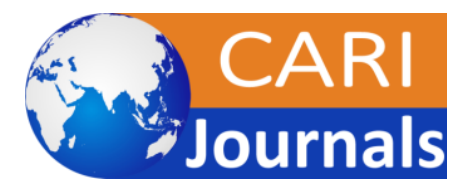

www.carijournals.org

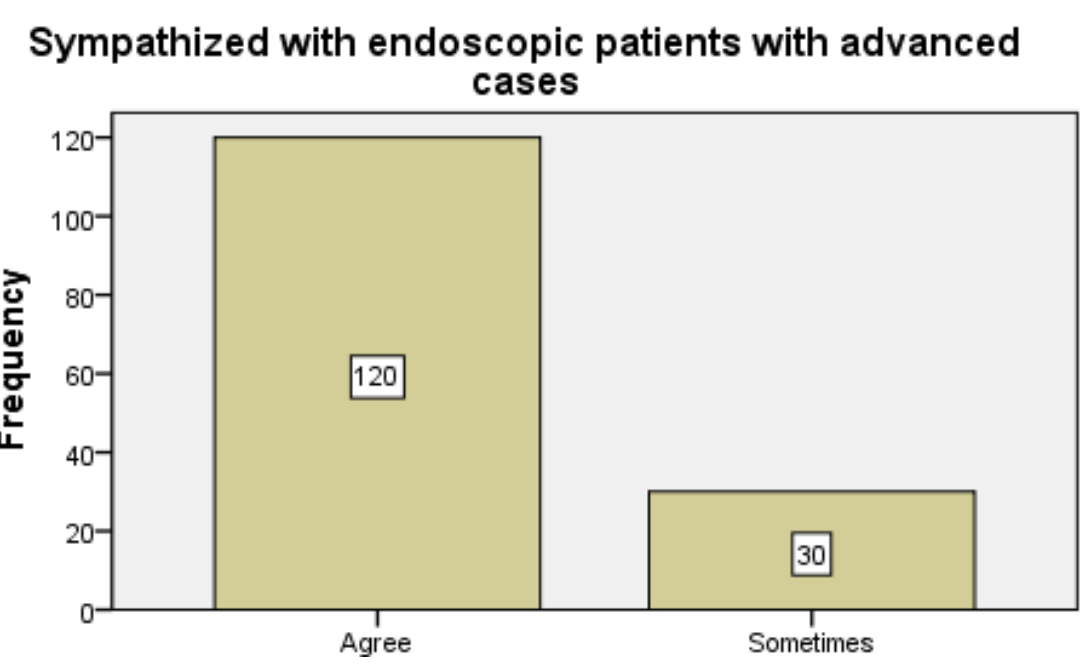

FIG 22

Figure 22 shows that many participants agreed to it that they show sympathy with endoscopic patient having advanced cases $(80 \%)$ but some of them answered sometimes $(\mathbf{2 0 \%})$.

\begin{tabular}{|l|r|r|r|r|}
\hline & Frequency & \multicolumn{1}{|c|}{ Percent } & \multicolumn{1}{c|}{$\begin{array}{c}\text { Valid } \\
\text { Percent }\end{array}$} & $\begin{array}{c}\text { Cumulative } \\
\text { Percent }\end{array}$ \\
\hline Agree & 121 & $80.7 \%$ & $80.7 \%$ & $80.7 \%$ \\
\hline Sometimes & 29 & $19.3 \%$ & $19.3 \%$ & $100 \%$ \\
\hline Total & 150 & $100 \%$ & $100 \%$ & \\
\hline
\end{tabular}

Table 23 
International Journal of Health, Medicine and Nursing Practice ISSN 2710-1150 (Online)

Vol. 3, Issue No. 2, pp 61-106, 2021

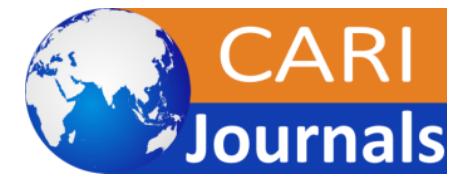

$\underline{\text { www.carijournals.org }}$

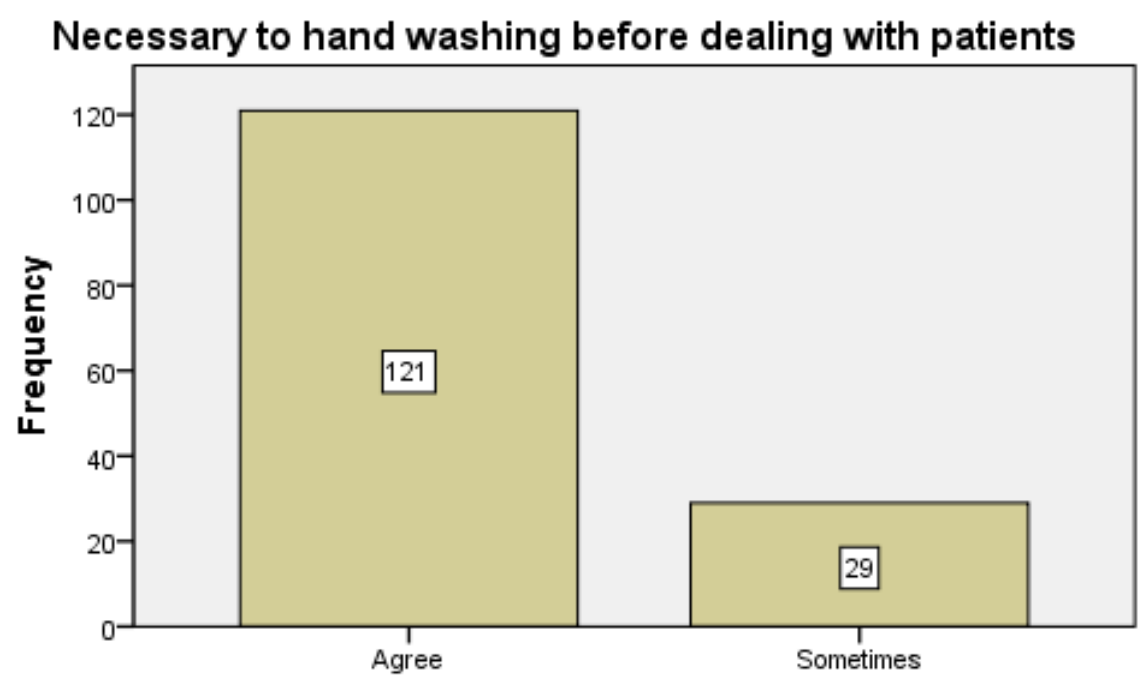

FIG 23

Figure 23 shows that many participants agreed to it that its necessary to wash hands before dealing with patients $(\mathbf{8 0 . 7 \%})$ some of them answered sometimes $(19.3 \%)$.

\begin{tabular}{|l|r|r|r|r|}
\hline & Frequency & \multicolumn{1}{|c|}{ Percent } & $\begin{array}{c}\text { Valid } \\
\text { Percent }\end{array}$ & $\begin{array}{c}\text { Cumulative } \\
\text { Percent }\end{array}$ \\
\hline Agree & 118 & $78.7 \%$ & $78.7 \%$ & $78.7 \%$ \\
\hline Sometimes & 14 & $9.3 \%$ & $9.3 \%$ & $88 \%$ \\
\hline Disagree & 18 & $12 \%$ & $12 \%$ & $100 \%$ \\
\hline Total & 150 & $100 \%$ & $100 \%$ & \\
\hline
\end{tabular}

Table 24 
International Journal of Health, Medicine and Nursing Practice ISSN 2710-1150 (Online)

Vol. 3, Issue No. 2, pp 61-106, 2021

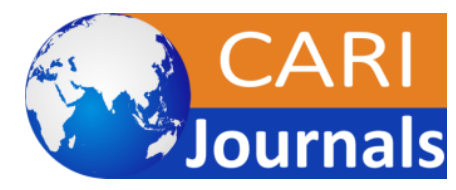

www.carijournals.org

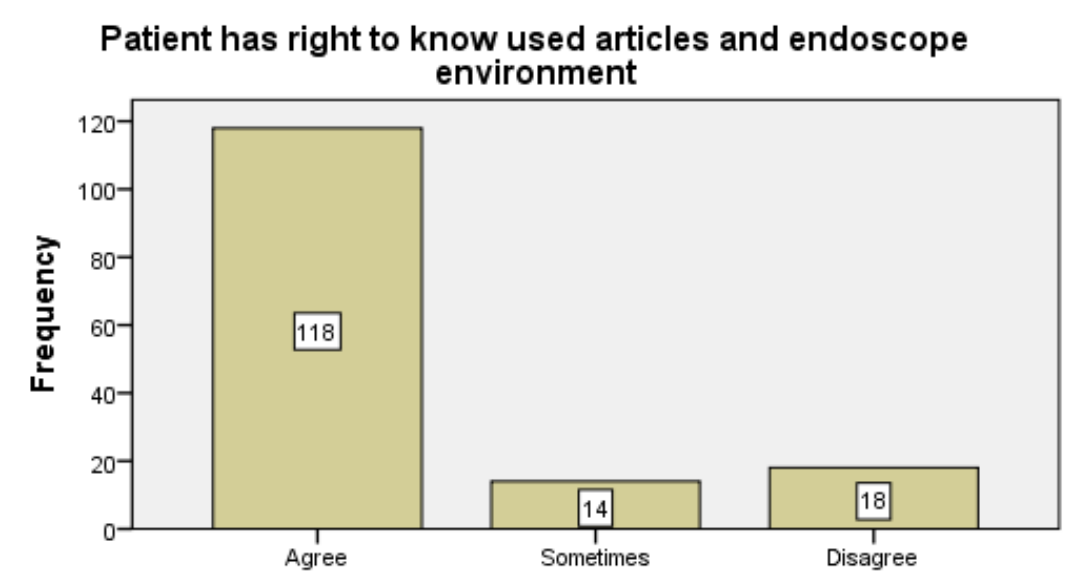

FIG 25

Figure 25 shows that many participants agreed to it that its patient right to know the used articles and endoscopy environment $\mathbf{( 7 8 . 7 \% )}$ ) some of them disagreed (12\%) while few answered sometimes $(9.3 \%)$.

\begin{tabular}{|l|r|r|r|r|}
\hline & Frequency & \multicolumn{1}{|c|}{ Percent } & $\begin{array}{c}\text { Valid } \\
\text { Percent }\end{array}$ & $\begin{array}{c}\text { Cumulative } \\
\text { Percent }\end{array}$ \\
\hline Agree & 114 & $76 \%$ & $76 \%$ & $76 \%$ \\
\hline Sometimes & 10 & $6.7 \%$ & $6.7 \%$ & $82.7 \%$ \\
\hline Disagree & 26 & $17.3 \%$ & $17.3 \%$ & $100 \%$ \\
\hline Total & 150 & $100 \%$ & $100 \%$ & \\
\hline
\end{tabular}

Table 26 
International Journal of Health, Medicine and Nursing Practice ISSN 2710-1150 (Online)

Vol. 3, Issue No. 2, pp 61-106, 2021

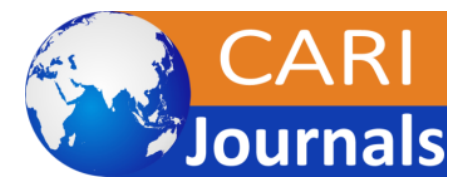

www.carijournals.org

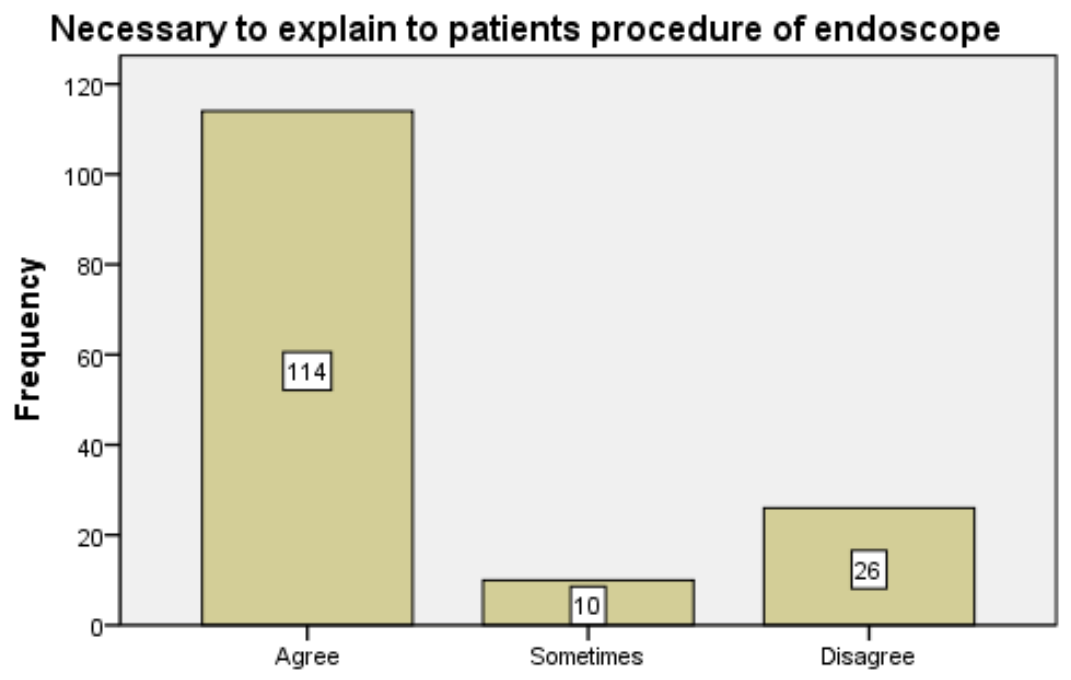

FIG 26

Figure 26 shows that many participants agreed to it that its patient right to know the used articles and endoscopy environment (76\%) some of them disagreed $(17.3 \%)$ while few answered sometimes $(6.7 \%)$.

\begin{tabular}{|l|r|r|r|r|}
\hline & Frequency & \multicolumn{1}{|c|}{ Percent } & \multicolumn{1}{c|}{$\begin{array}{c}\text { Valid } \\
\text { Percent }\end{array}$} & $\begin{array}{c}\text { Cumulative } \\
\text { Percent }\end{array}$ \\
\hline Sometimes & 49 & $32.7 \%$ & $32.7 \%$ & $32.7 \%$ \\
\hline Disagree & 101 & $67.3 \%$ & $67.3 \%$ & $100 \%$ \\
\hline Total & 150 & $100 \%$ & $100 \%$ & \\
\hline
\end{tabular}

Table 27 
International Journal of Health, Medicine and Nursing Practice ISSN 2710-1150 (Online)

Vol. 3, Issue No. 2, pp 61-106, 2021

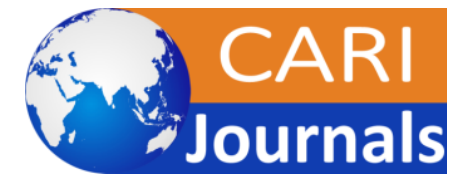

www.carijournals.org

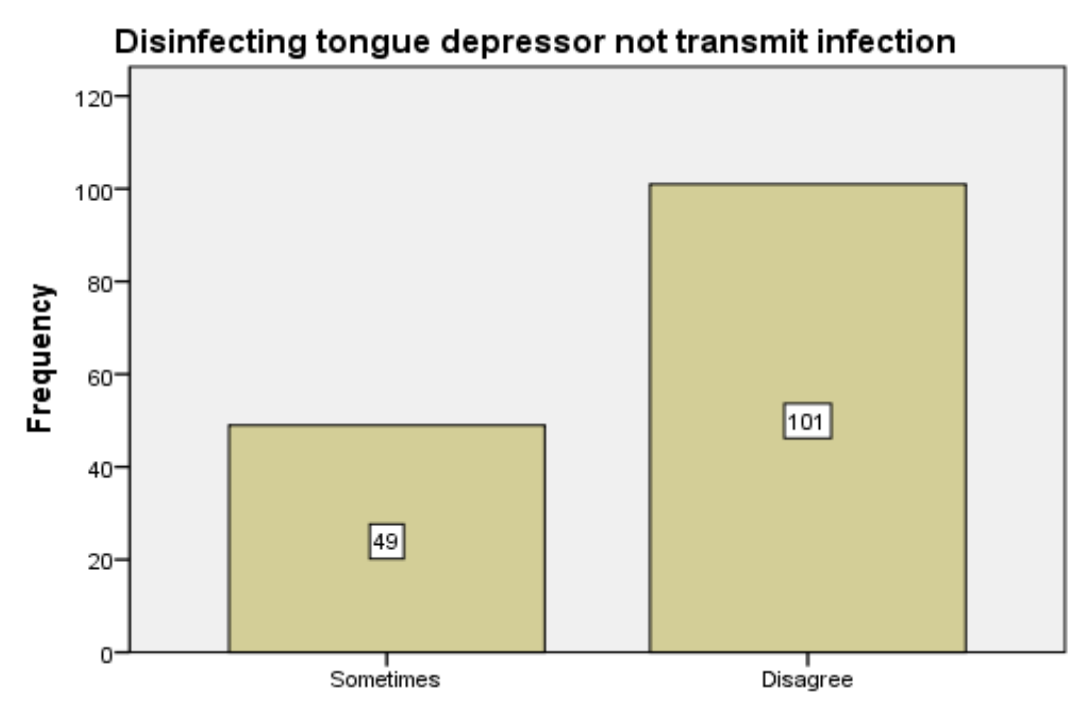

FIG 27

Figure 27 shows that many participants disagreed to it that disinfecting tongue depressors not transmit infections (67.3\%) while other answered it as sometimes (32.7\%).

\begin{tabular}{|l|r|r|r|r|}
\hline & Frequency & \multicolumn{1}{|c|}{ Percent } & $\begin{array}{c}\text { Valid } \\
\text { Percent }\end{array}$ & $\begin{array}{c}\text { Cumulative } \\
\text { Percent }\end{array}$ \\
\hline Agree & 130 & $86.7 \%$ & $86.7 \%$ & $86.7 \%$ \\
\hline Sometimes & 20 & $13.3 \%$ & $13.3 \%$ & $100 \%$ \\
\hline Total & 150 & $100 \%$ & $100 \%$ & \\
\hline
\end{tabular}

Table 28 
International Journal of Health, Medicine and Nursing Practice ISSN 2710-1150 (Online)

Vol. 3, Issue No. 2, pp 61-106, 2021

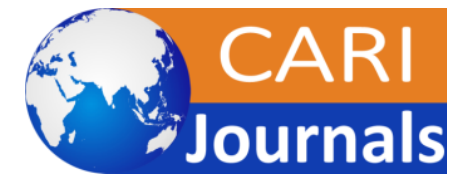

$\underline{\text { www.carijournals.org }}$

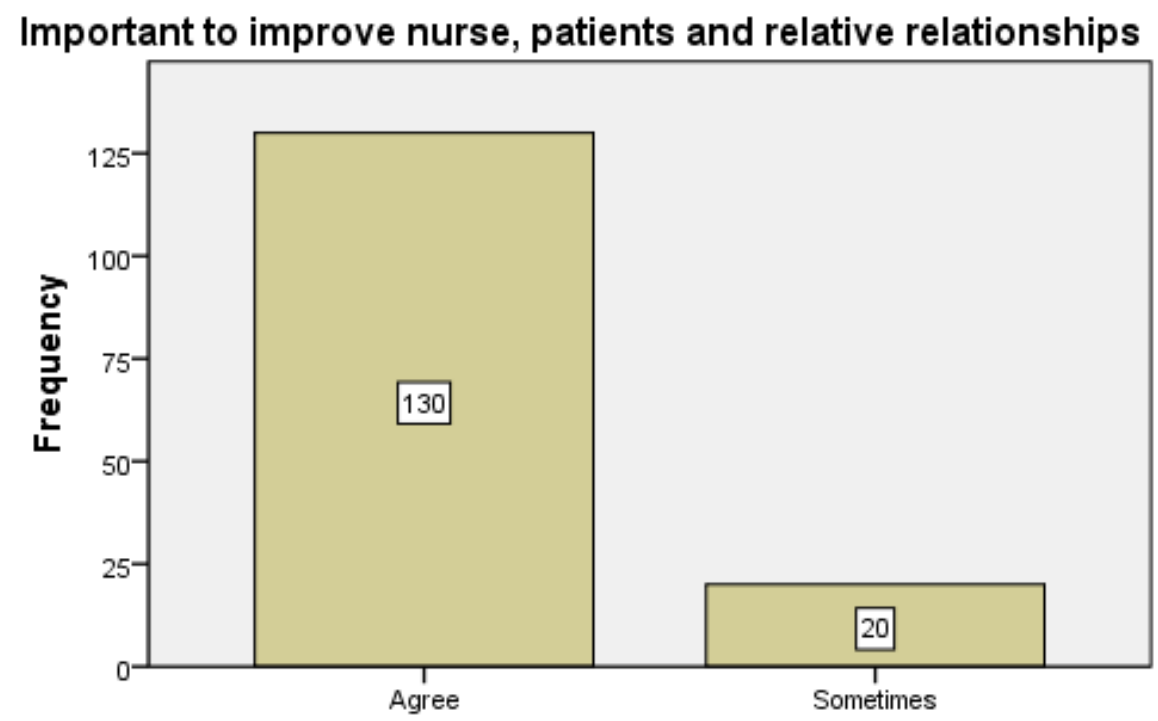

FIG 28

Figure 28 shows that many participants agreed to it that its important to improve nure, patient and relative relationships $(\mathbf{8 6 . 7 \%})$ while few answered sometimes $(\mathbf{1 3 . 3 \%})$.

\begin{tabular}{|l|r|r|r|r|}
\hline & Frequency & Percent & $\begin{array}{c}\text { Valid } \\
\text { Percent }\end{array}$ & $\begin{array}{c}\text { Cumulative } \\
\text { Percent }\end{array}$ \\
\hline Agree & 116 & $77.3 \%$ & $77.3 \%$ & $77.3 \%$ \\
\hline Sometimes & 34 & $22.7 \%$ & $22.7 \%$ & $100 \%$ \\
\hline Total & 150 & $100 \%$ & $100 \%$ & \\
\hline
\end{tabular}

Table 29 
International Journal of Health, Medicine and Nursing Practice ISSN 2710-1150 (Online)

Vol. 3, Issue No. 2, pp 61-106, 2021

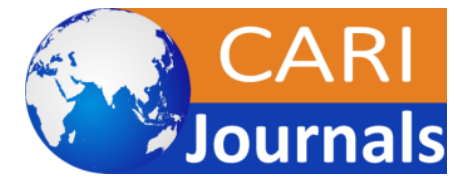

$\underline{\text { www.carijournals.org }}$

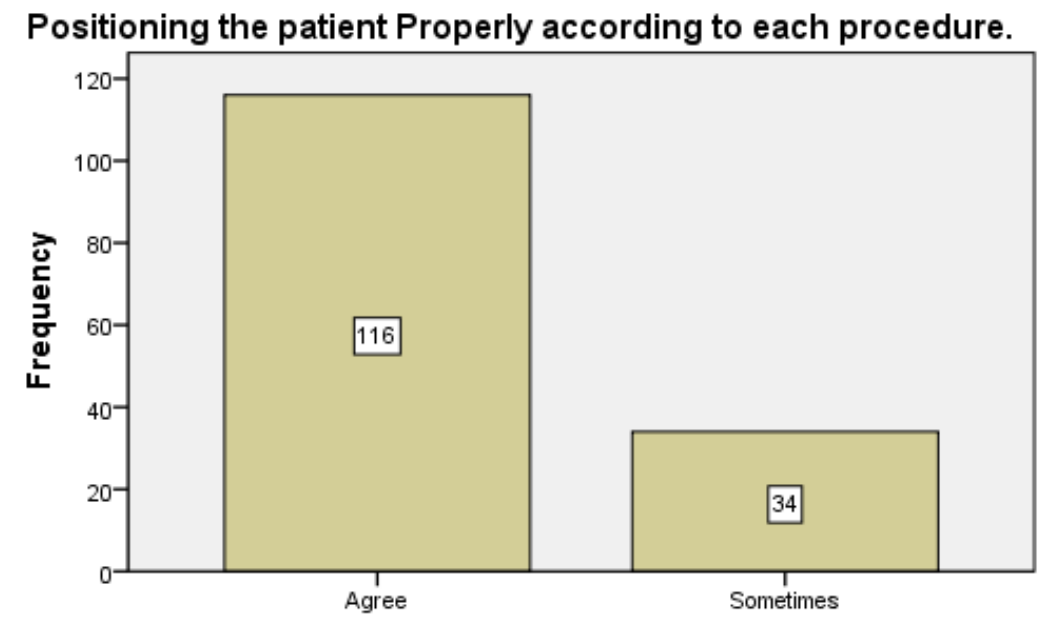

FIG 29

Figure 29 shows that many participants agreed to it that its essential to position the patient properly to each procedure undergone $\mathbf{( 7 7 . 3 \%}$ ) while few answered sometimes $(\mathbf{2 2 . 7 \%})$.

\begin{tabular}{|l|r|r|r|r|}
\hline & Frequency & Percent & $\begin{array}{c}\text { Valid } \\
\text { Percent }\end{array}$ & $\begin{array}{c}\text { Cumulative } \\
\text { Percent }\end{array}$ \\
\hline Agree & 123 & $82 \%$ & $82 \%$ & $82 \%$ \\
\hline Sometimes & 12 & $8 \%$ & $8 \%$ & $90 \%$ \\
\hline Disagree & 15 & $10 \%$ & $10 \%$ & $100 \%$ \\
\hline Total & 150 & $100 \%$ & $100 \%$ & \\
\hline
\end{tabular}


International Journal of Health, Medicine and Nursing Practice ISSN 2710-1150 (Online)

Vol. 3, Issue No. 2, pp 61-106, 2021

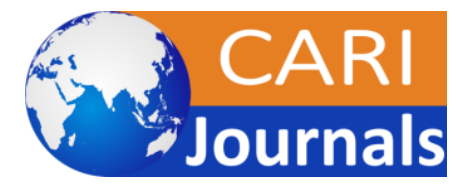

$\underline{\text { www.carijournals.org }}$

Table 30

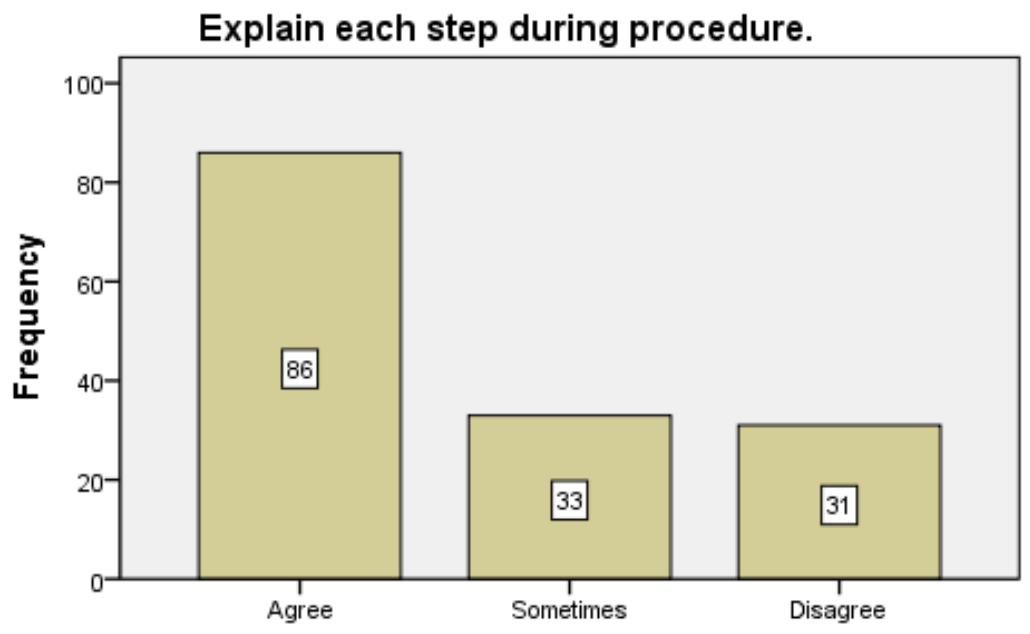

FIG 30

Figure 30 shows that many participants agreed to it that its important to explain each step during procedure $(82 \%)$ some of them disagreed $(10 \%)$ while few answered sometimes $(8 \%)$.

\begin{tabular}{|l|r|r|r|r|}
\hline & Frequency & Percent & $\begin{array}{c}\text { Valid } \\
\text { Percent }\end{array}$ & $\begin{array}{c}\text { Cumulative } \\
\text { Percent }\end{array}$ \\
\hline Agree & 114 & $76 \%$ & $76 \%$ & $76 \%$ \\
\hline Sometimes & 33 & $22 \%$ & $22 \%$ & $98 \%$ \\
\hline Disagree & 3 & $2 \%$ & $2 \%$ & $100 \%$ \\
\hline Total & 150 & $100 \%$ & $100 \%$ & \\
\hline
\end{tabular}

Table 31 
International Journal of Health, Medicine and Nursing Practice ISSN 2710-1150 (Online)

Vol. 3, Issue No. 2, pp 61-106, 2021

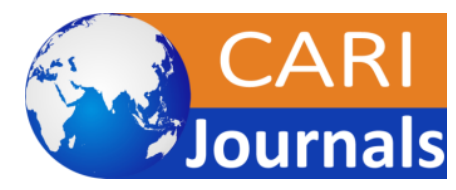

$\underline{\text { www.carijournals.org }}$

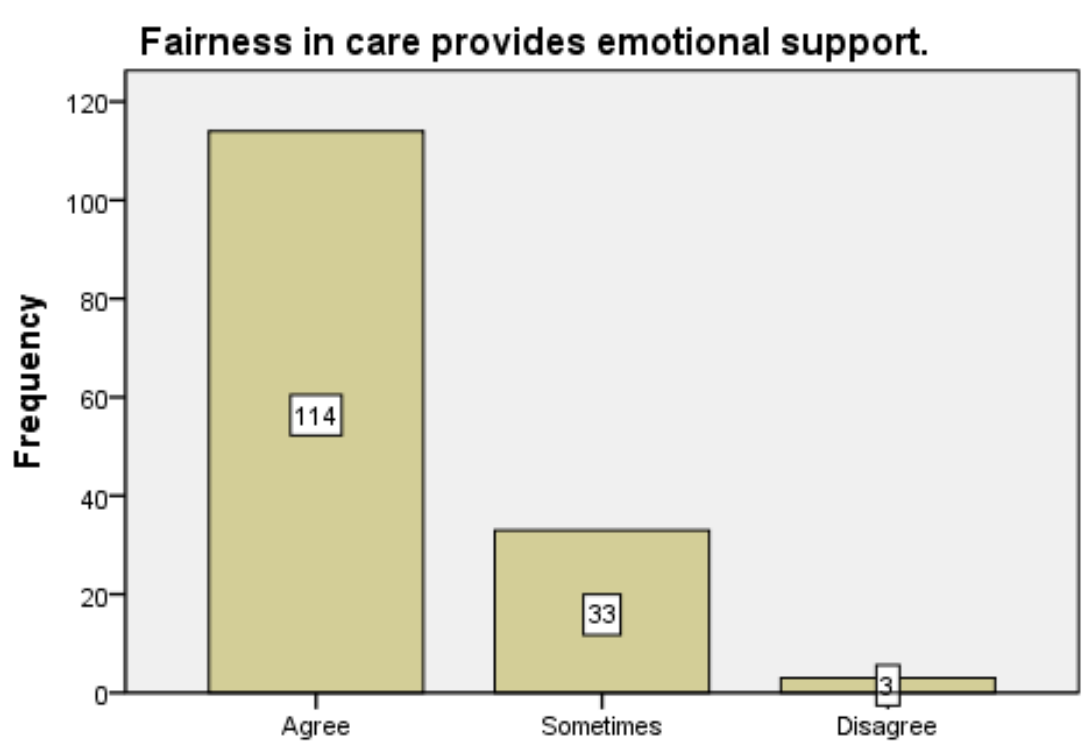

FIG 31

Figure 31 shows that many participants agreed to it that its fairness in care provide emotional support to patient $\mathbf{( 7 6 \% )}$ ) while several answered sometimes $(22 \%)$ but few disagreed to it $(2 \%)$.

\begin{tabular}{|l|r|r|r|r|}
\hline & Frequency & \multicolumn{1}{|c|}{ Percent } & \multicolumn{1}{|c|}{$\begin{array}{c}\text { Valid } \\
\text { Percent }\end{array}$} & $\begin{array}{c}\text { Cumulative } \\
\text { Percent }\end{array}$ \\
\hline Agree & 119 & $79.3 \%$ & $79.3 \%$ & $79.3 \%$ \\
\hline Sometimes & 31 & $20.7 \%$ & $20.7 \%$ & $100 \%$ \\
\hline Total & 150 & $100 \%$ & $100 \%$ & \\
\hline
\end{tabular}

Table 32 
International Journal of Health, Medicine and Nursing Practice ISSN 2710-1150 (Online)

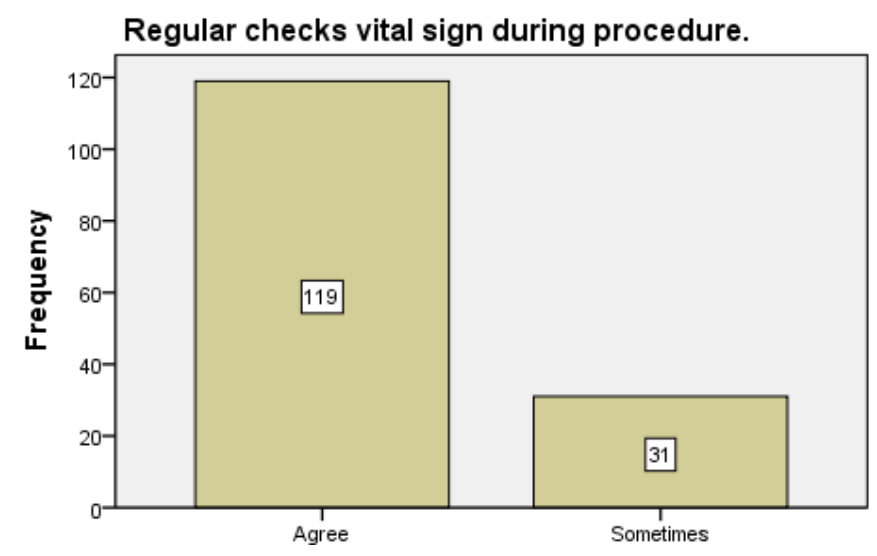

FIG 32

Figure 32 shows that many participants agreed to it that its essential regularly check vital signs during procedure $(\mathbf{7 9 . 3 \%})$ while few answered sometimes $(\mathbf{2 0 . 7 \%})$.

\begin{tabular}{|l|r|r|r|r|}
\hline & Frequency & \multicolumn{1}{|c|}{ Percent } & $\begin{array}{c}\text { Valid } \\
\text { Percent }\end{array}$ & \multicolumn{1}{c|}{$\begin{array}{c}\text { Cumulative } \\
\text { Percent }\end{array}$} \\
\hline Agree & 133 & $88.7 \%$ & $88.7 \%$ & $88.7 \%$ \\
\hline Sometimes & 17 & $11.3 \%$ & $11.3 \%$ & $100 \%$ \\
\hline Total & 150 & $100 \%$ & $100 \%$ & \\
\hline
\end{tabular}


International Journal of Health, Medicine and Nursing Practice ISSN 2710-1150 (Online)

Vol. 3, Issue No. 2, pp 61-106, 2021

Table 33

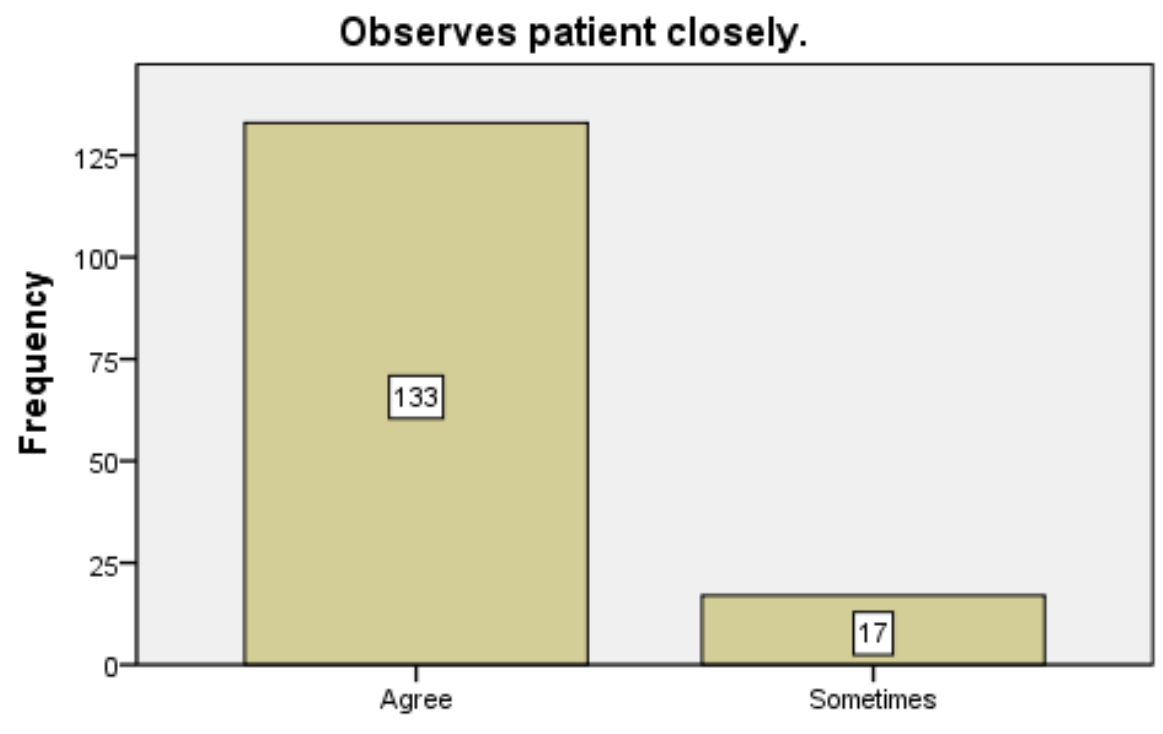

FIG 33

Figure 33 shows that many participants agreed to it that its essential observe patient closely $(\mathbf{8 8 . 7 \%})$ while few answered sometimes $(\mathbf{1 1 . 3 \%})$.

\begin{tabular}{|l|r|r|r|r|}
\hline & Frequency & \multicolumn{1}{|c|}{ Percent } & $\begin{array}{c}\text { Valid } \\
\text { Percent }\end{array}$ & \multicolumn{1}{c|}{$\begin{array}{c}\text { Cumulative } \\
\text { Percent }\end{array}$} \\
\hline Agree & 128 & $85.3 \%$ & $85.3 \%$ & $85.3 \%$ \\
\hline Sometimes & 22 & $14.7 \%$ & $14.7 \%$ & $100 \%$ \\
\hline Total & 150 & $100 \%$ & $100 \%$ & \\
\hline
\end{tabular}


International Journal of Health, Medicine and Nursing Practice ISSN 2710-1150 (Online)

Vol. 3, Issue No. 2, pp 61-106, 2021

Table 34

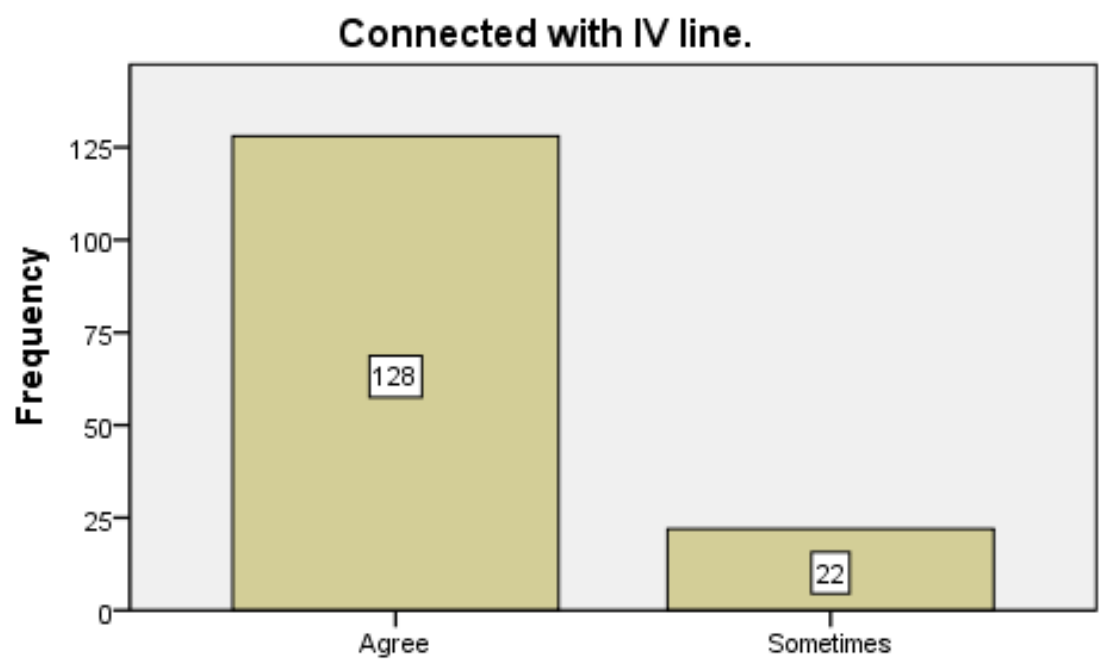

FIG 34

Figure 34 shows that many participants agreed to it that its essential to check whether patient is connected with IV line $(\mathbf{8 5 . 3 \%})$ while few answered sometimes $(\mathbf{1 4 . 7 \%})$.

\begin{tabular}{|l|r|r|r|r|}
\hline & Frequency & \multicolumn{1}{|c|}{ Percent } & $\begin{array}{c}\text { Valid } \\
\text { Percent }\end{array}$ & $\begin{array}{c}\text { Cumulative } \\
\text { Percent }\end{array}$ \\
\hline Agree & 85 & $56.7 \%$ & $56.7 \%$ & $56.7 \%$ \\
\hline Sometimes & 63 & $42 \%$ & $42 \%$ & $98.7 \%$ \\
\hline Disagree & 2 & $1.3 \%$ & $1.3 \%$ & $100 \%$ \\
\hline Total & 150 & $100 \%$ & $100 \%$ & \\
\hline
\end{tabular}

Table 35 
International Journal of Health, Medicine and Nursing Practice ISSN 2710-1150 (Online)

Vol. 3, Issue No. 2, pp 61-106, 2021

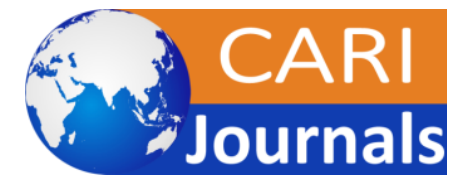

www.carijournals.org

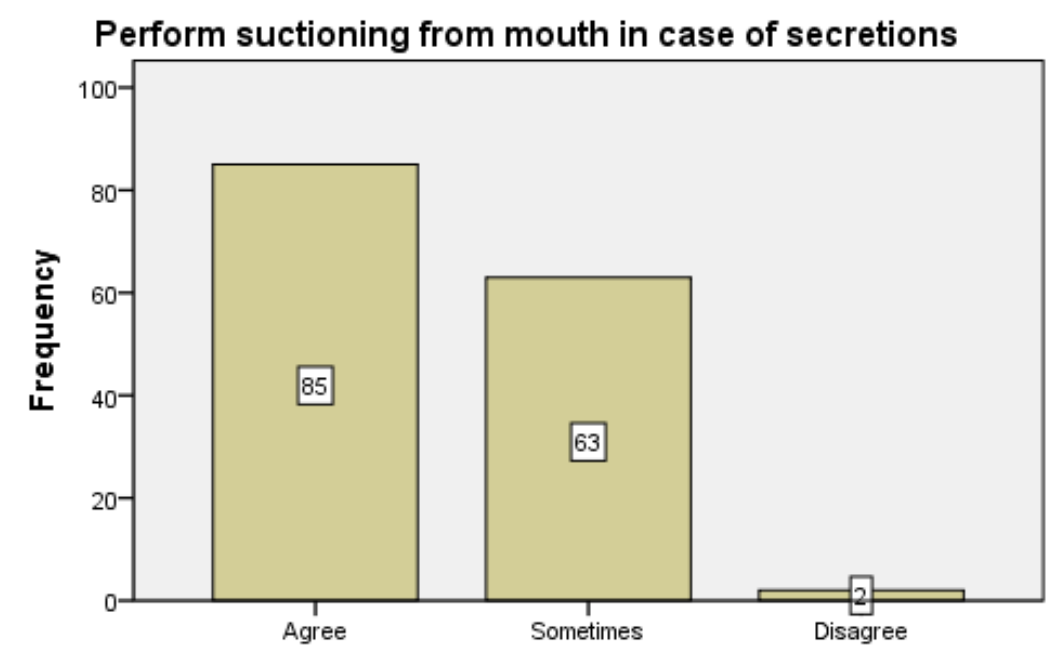

FIG 35

Figure 35 shows that many participants agreed to it that its essential to perform suctioning from mouth in case of secretions $(\mathbf{5 6 . 7 \%})$ while many of them answered sometimes $(\mathbf{4 2 \%})$ but few disagreed to it $(\mathbf{1 . 3 \%})$.

\begin{tabular}{|l|r|r|r|r|}
\hline & Frequency & Percent & $\begin{array}{c}\text { Valid } \\
\text { Percent }\end{array}$ & $\begin{array}{c}\text { Cumulative } \\
\text { Percent }\end{array}$ \\
\hline Agree & 150 & $100 \%$ & $100 \%$ & $100 \%$ \\
\hline Total & 150 & $100 \%$ & $100 \%$ & \\
\hline
\end{tabular}

Table 36 


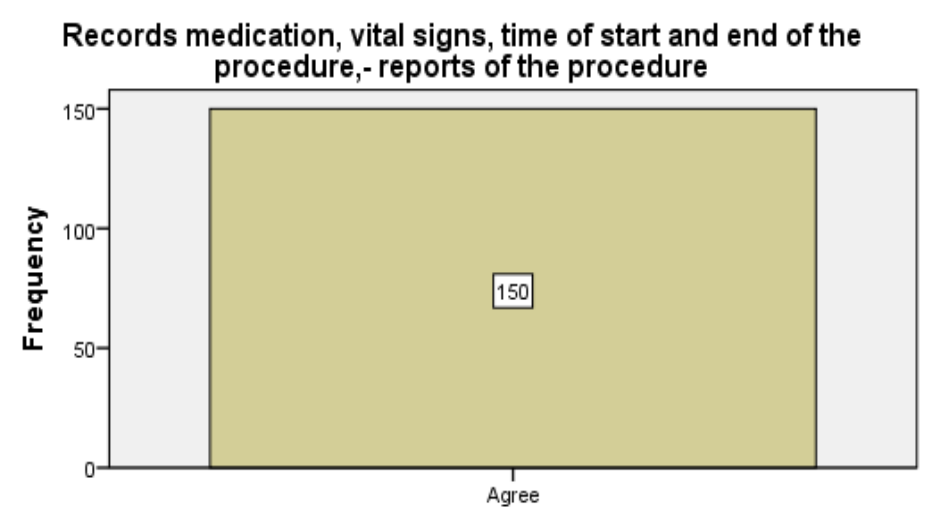

FIG 36

Figure 36 shows that all participants agreed to it that its essential to record medication, vital signs from start to the end of procedure and reports of procedure $(\mathbf{1 0 0 \%})$

\section{Results}

Data was collected from total 150 nurses of Nishtar Hopital Multan majority $n=84$ were females (56\%). Majority $\mathrm{n}=57$ age group of them was less than 45 years (38\%). Majority $\mathrm{n}=120$ qualification of them was diploma degree $(80 \%)$. Majority $n=123$ or them have work duration equals to 8 years $(82 \%)$. Majority $n=109$ of endoscopy experience of participants was less than 10 years $(72.7 \%)$. Majority $n=123$ of them received training for endoscopy procedures $(82 \%)$. Majority $n=123$ of them have received vaccination of $\mathrm{HBV}(82 \%)$. Majority $n=150$ of them agreed that hand washing after toilet is important $(100 \%)$. Majority $n=150$ of them agreed to it that hand washing before drinking and eating is necessary $(100 \%)$. Majority $n=133$ of them agreed that hand washing after attending each patient is very essential (88.7\%). Majority $n=150$ of participants agreed that hand washing after each endoscopy is very necessary (100\%).

Majority $\mathrm{n}=131$ of them agreed that nurses and doctors can transmit infection to the patients $(87.3 \%)$. Majority $\mathrm{n}=124$ of them agreed that doctors and nurses should wear masks during endoscopy $(82.7 \%)$. Majority $\mathrm{n}=131$ of them agreed that doctors and nurses should wear gowns during endoscopy $(87.3 \%)$. Majority $\mathrm{n}=129$ of them agreed that doctors and nurses should wear surgical gloves during endoscopy (87.3\%) Majority $n=144$ of them agreed that eating and drinking should be prohibited in endoscopy (96\%). Majority $n=150$ of them agreed that smoking should be restricted in endoscopy (100\%). Majority $n=44$ of them agreed to it that its important to provide comfortable place to patient $(56 \%)$. Majority $n=62$ of them disagreed that patient do not effects psychological state $(41.3 \%)$. Majority $n=88$ of them disagreed to that fairness of endoscopic patients blocks delay in providing nursing care to them (58.7\%). Majority $n=88$ of them agreed hand washing only kills organisms $(58.7 \%)$. Majority $\mathrm{n}=120$ of them agreed that showing sympathy to endoscopic patient with advance cases is very important $(80 \%)$. Majority $\mathrm{n}=121$ agreed it is important to hand wash before dealing with patients $(80.7 \%)$. Majority $n=118$ of them agreed that patient has right to know the used articled and endoscopy environment (78.7\%). Majority $n=114$ of them agreed that it is important to explain the procedure of endoscopy to patient (76\%). Majority $\mathrm{n}=101$ of them disagreed that disinfecting tongue depressors do not spread 
infection $(67.3 \%)$. Majority $n=130$ of them agreed that it is important to improve nurses, patients, and relative relationship ( $86.7 \%$ ). Majority $n=116$ of them agreed that it is important to position patient properly according to each procedure $(77.3 \%)$. Majority $n=123$ of them agreed that it is important to explain each step during procedure $(82 \%)$. Majority $n=114$ of them agreed that fairness in care of patients provides emotional support to them $(76 \%)$. Majority $n=133$ of them agreed that it is important to check vital signs during procedure (79.3\%). Majority $\mathrm{n}=128$ of them agreed that its important to check whether patient is connected with IV line (85.3\%). Majority $\mathrm{n}=85$ agreed that perform suctioning from mouth in case of secretions $(56.7 \%)$. Majority $n=150$ of them agreed that its important to record medications, vital signs from start till end of procedure and to record the reports of procedure (100\%).

\section{DISSCUSSION}

Endoscopy nurses play a important role within the provision of safe, prime quality endoscopy. Nurses have several tasks. Prepare the scrutiny space with the proper instrument and necessary devices for examination of the higher or lower GI tract, is extremely important. it's additionally crucial that the nurse gives the proper information concerning the procedure to the patient, to alleviate anxiety and to give explanations about the modality of the scrutiny procedure. throughout the procedure the nurse must facilitate the endoscopist and, once indicated, the anesthesist. when the completion of the procedure, the nurse should carry-on with the reprocessing of the endoscopic instrument and of the devices.

Present study was conducted to assess the knowledge, attitudes and practices among nurses of Nishtar Hopital Multan. Several studies were conducted related to this topic but the current study showed different results. In past conducted study of Amer et al. majority of nurses were females and this presently conducted study also revealed similar results that large percentage of females were participants of this study. Moreover, again a similarity emerge that $80 \%$ of nurses in past study were diploma holder same in our study $80 \%$ of female nurses have qualification status as diploma education. But in study of Amer et al. nurses have endoscopic work experience equals to 8-28 years but in this present study nurses showed different results majority of them have 8 years experience. In past conducted study only two fifth of nurses received special training on endoscopy but in our study all of them have received appropriate endoscopy training. In addition in past studies only four fifth of nurses received vaccinations while this present study showed that majority of them have received vaccination HBV. In past studies four fifth of nurses has satisfactory knowledge related to endoscopy precautions and measures while in our present study knowledge of nurses by having appropriate training is improved optimistically to higher percentage In past study of Amer et al. poor knowledge of nurses in baseline observed while in present study this loop hole is minimized. Present study showed similar results to the Bertleff's et al. who noticed that after training or education the knowledge results in progressive practice of nurses. Ramsey and his colleague based of their have a look at that, maximum of the nursesdid now no longer acquire any unique training or Inservice education approximately endoscope reprocessing practices. Most of the authors stated that training and education, which include competency testing, as a minimum annually. This facilitates professional nurse to maintain updated at the maximum recent traits in nursing and to have the ability to control the needs of nursing practice. Educational application and education guides are additives of workforce 
improvement. It is advocated that non-stop training in nursing is wanted to sell improvement of expertise, abilities and attitudes of nurses and to enhance the nice of care given for their patients. Also the fashioned education guides played an essential position in improving and updating nurses expertise and performance.

\section{CONCLUSION}

Finally we conclude that the nurse age, training and dealing period might have an effect on the level of nurses data relating to canal endoscopy including; general precautions, basic steps to wash and medical care in examination unit. The majority of nurses had positive attitude. While coaching and qualification can affect dealing with the patients additionally to nurses level of apply before, throughout and manual disinfecting of endoscopy. So, we tend to recommend adequate education and training of all nurses working with gastrointestinal endoscopy unit, with continuous analysis of nurses work practice. Periodic evaluation might indicate interference with training programs ought to be enclosed both theoretical and practical. more studies are necessary to spot effects of educational programs on nurses performance in endoscopy unit.

Extensive training of staff involved in endoscope reprocessing is mandatory for quality assurance and for effective infection control and documentation of this taring is required. The efficacy of manual cleaning and HLD operator dependent, thus assignment of personnel responsible for endoscope. Suggests the importance of using standard clinical guidelines and approaches to prepare educational curriculum, internship education in hospital, and educational courses in hospital.Guideline for HCWs especially radiology staff and health care policy makers.

\section{ACKNOWLEDGEMENT}

Firstly, I am very thankful to Allah Almighty for providing me an opportunity and strength to accomplish my research work. This study is dedicated to my family, teachers and colleagues. I would like to heartedly thanks my respected teachers especially Mam Kousar Parveen (Assistant Professor in Lahore School of Nursing UOL) without her support I will never be capable to complete this research. And a very special thanks to the respected and ideal personality of our department our principal Mr. Muhammad Afzal (Head of Department in Lahore School of Nursing UOL) for supporting me and encouraging me.

\section{References}

Alfa MJ, Olson N , Degagne P(2016). The Reliance Endoscope Processing System. AJIC 2016, 34:561

Ali R. Hamed Shehata (2013). Thesis; Endoscopy and Gastroenterology Department, Assiut university 2013.

Bertleff M, Helm JA, Bemelman WA, Vandeham AC, Vandeharst E, Oei HI (2019). Randomized Clinical Trial of Endoscopy: The LAMA Trial Randomized Clinical Trial of

Endoscopy procedures: The LAMA Trial. World Journal of Surgery 2019;33 (7): 1368-1377.

California Department of Health Services (CDHS).State Health Department Releases Revised Nurse To-Patient ratios for public comment on endoscopy 2003. 
International Journal of Health, Medicine and Nursing Practice

ISSN 2710-1150 (Online)

Vol. 3, Issue No. 2, pp 61-106, 2021

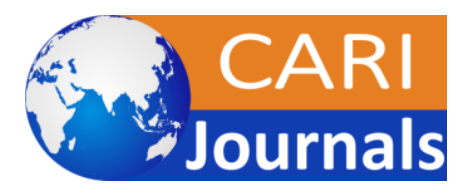

www.carijournals.org

Carl J. Alvarado, Mark R (2019). APIC Guideline for infection prevention and control Flexible Endoscopy. Am J Infect Control 2018.

Canadian Standards Association. Decontamination of Reusable Medical Devices during endoscopy. Mississauga, Ontario; 2018.

Cotton PB, Williams CB, Hawes RH, Saunders BP (2018). Practical Gastrointestinal Endoscopy, the Fundamentals, Philadelphia: Lippincott Williams and Wilkins, 2018. Sixth edition, chapter 3, p.2.

Cowen AE (2014). The clinical risks of infection associated with endoscopy. Can J Gastroenterol 2014.

Day T, Wainwright SP, Wilson-Barnett J (2015). An evaluation of a teaching intervention to Improve The practice of endoscopic procedures in intensive care units. J Clin Nurs. 2015 Sep;10 (5):682-96.

El-Shamaa ET (2010). Developing A Control Action Plan For Infection Prevention At The Endoscopy Unit. International Journal Of Academic Research 2010, 2(4):

Gómez M, Llach J (2013). Role of nurses in endoscopy. 2013.

Kennedy J, Oconnor P and Sadovnick AD (2016). Multiple methods of endoscopy influenced By patient requirements: American Journal of Nursing 2016.

Majeski J, Lynch W, Durst G (2019). EXPLANATION OF ENDOSCOPY. Am J Surg. 2019 Nov;198(5).

Mehta AC, Prakash UBS, Garland R, et al (2015). American College of physicians and American Association for endoscopy Consensus Statement: Prevention of Flexible EndoscopyAssociated Infection. 2015; 128:1742-55.

Mohamad HAE-RAE-A, Mohamad ZA, EL-Abdeen Z, Sayed A, editors. impact of designed nursing teaching protocol on nurses performance and patients out comes 2014.

Nelson DB (2016). Recent advances in epidemiology and prevention of endoscopic related infections, Current Opinions in Infectious Diseases 2016.

Pathmakanthan S, Murray I, Smith K, Heeley R, Donnelly M (2016). Nurse Endoscopists in United Kingdom health care: a survey of prevalence, skills and attitudes. J Adv Nurs 2016; 36: 705-10.

Paudyal P, Simkhada P, Bruce J (2018). Knowledge, attitude, and practice regarding endoscopy procedures among Neplease health care workers. Am J Infect Control 2018, 36:595-597.

Petersen BT, Chennat J, Cohen J, Cotton PB, Greenwald DA, Kowalski TE et al (2019). ASGE Quality Assurance in Endoscopy Committee, Rutala WA; Society for Healthcare Epidemiology of America.Multisociety guideline on reprocessing flexible GI endoscopes: 2019.

Public Health Agency of Canada. Infection Prevention and Control Guideline for Flexible 
International Journal of Health, Medicine and Nursing Practice

ISSN 2710-1150 (Online)

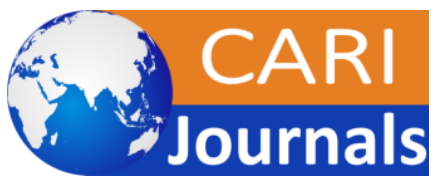

Vol. 3, Issue No. 2, pp 61-106, 2021

www.carijournals.org

Endoscopy (2016).

Robinson J, Moreau D, McCann J (2015). Hand Book of Medical-Endoscopic Nursing , $3^{\text {rd }}$ ed.,Pennsylvania:Springhouse 2015, pp. 303-310.

Reda AA, Vandeweerd JM, Syre TR, Egata G (2019). Experiences of healthcare workers towards endoscopy in Ethiopia: attitudes toward universal precautions during endoscopy. J Hosp Infect 2019, 7: 163-169.

Salvetto M (2017). The nurse's role during endoscopic procedures. Minerva Med 2017.

Smith, NE , Timby, BK (2018). Introductory medical endoscopy nursing, Caring for clients Undergoing endoscopy, 10th ed. Philadelphia, Wolters Kluwer Health, Lippincott Williams \& Wilkins 2018, Pp (583- 585).

Sagoe-Moses C, Pearson RD, Perry J, Jagger J(2016). Risks to patients during endoscopy. N Engl J Med 2016.

Stein AD, Makarawo TP, Ahmad MF (2017). A survey of doctors' and nurses knowledge, attitudes and compliance with infection control guidelines during Endoscopy in Birmingham teaching hospitals. J Hosp Infect 2017.

Saniee M, shalmanie HM, Zali MR. knowledge, attitudes and practices of GI and endoscopy nurses in iran. How much does nurses know about modes of transmission and prevention of HIV / AIDS ? : 729 offfical journal of the Amercian College of Gastroenterology I ACG. 2004, 99:S236.

Weber DJ, Rutala WA (2012). Lessons from outbreaks associated with endoscopy. J Hosp Infect 2012.

Vaessen HH, Knape JT (2016). Considerable Variability of Procedural Sedation and Analgesia Practices for Gastrointestinal Endoscopic Procedures in Europe. Clin Endosc, 2016, 49(1):47-55.

Vergis AS, Thomson D , Pieroni P, Dhalla S (2017). Reprocessing Flexible Gastrointestinal Endoscopies: Is It Necessary? .Endoscopy 2017; 39:737-9 